\title{
Topological Analysis of Chaotic Transport Through a Ballistic Atom Pump
}

Tommy A. Byrd

William \& Mary

John B. Delos

William \& Mary, jbdelos@wm.edu

Follow this and additional works at: https://scholarworks.wm.edu/aspubs

Part of the Physics Commons

\section{Recommended Citation}

Byrd, Tommy A. and Delos, John B., Topological Analysis of Chaotic Transport Through a Ballistic Atom Pump (2014). Physical Review E, 89(2).

https://doi.org/10.1103/PhysRevE.89.022907

This Article is brought to you for free and open access by the Arts and Sciences at W\&M ScholarWorks. It has been accepted for inclusion in Arts \& Sciences Articles by an authorized administrator of W\&M ScholarWorks. For more information, please contact scholarworks@wm.edu. 


\title{
Topological analysis of chaotic transport through a ballistic atom pump
}

\author{
Tommy A. Byrd and John B. Delos \\ Department of Physics, College of William and Mary, Williamsburg, Virginia 23187, USA
}

(Received 18 October 2013; published 10 February 2014)

\begin{abstract}
We examine a system consisting of two reservoirs of particles connected by a channel. In the channel are two oscillating repulsive potential-energy barriers. It is known that such a system can transport particles from one reservoir to the other, even when the chemical potentials in the reservoirs are equal. We use computations and the theory of chaotic transport to study this system. Chaotic transport is described by passage around or through a heteroclinic tangle. Topological properties of the tangle are described using a generalization of homotopic lobe dynamics, which is a theory that gives some properties of intermediate-time behavior from properties of short-time behavior. We compare these predicted properties with direct computation of trajectories.
\end{abstract}

DOI: 10.1103/PhysRevE.89.022907

PACS number(s): 05.45.Df, 05.60.Cd, 05.45.Ac, 37.10.Vz

\section{INTRODUCTION}

A ballistic atom pump is a system containing two or more reservoirs of neutral atoms or molecules and channels connecting them containing time-dependent potentials. "Ballistic" means that atoms move through the pump as independent particles, interacting only with the walls and with the timedependent potential. The pump we consider in this paper is one-dimensional; it has two reservoirs, particles approach from the left, and they are either transmitted through the pump to the right or reflected back to the left. The Hamiltonian is

$$
H(x, p)=\frac{p^{2}}{2}+V(x, t),
$$

where

$$
\begin{aligned}
V(x, t)= & U_{0}[1+\alpha \cos (\omega t)] e^{-(x+\hat{x})^{2} / 2 \sigma^{2}} \\
& +U_{0}[1+\alpha \cos (\omega t+\phi)] e^{-(x-\hat{x})^{2} / 2 \sigma^{2}} .
\end{aligned}
$$

This potential energy $V(x, t)$ has two repulsive Gaussian barriers centered at $\pm \hat{x}$; they have the same average height $U_{0}$, and they oscillate with the same amplitude $\alpha$ and frequency $\omega$, but they are $\phi=\pi / 2$ out of phase with each other.

There are two different motivations behind this work. (1) Similar pumps have been proposed for pumping electrons through mesoscopic junctions, a phenomenon called "quantum pumping." Such experiments are very difficult, so Das et al. $[1,2]$ suggested that ultracold neutral atoms might provide a suitable laboratory model for learning about the properties of such pumps. In Ref. [3], we studied scattering from a single barrier of such a pump. (2) When we examine particle trajectories through such pumps, we find that these systems are very nice models of chaotic transport [4-36].

This paper focuses on the chaotic-transport aspect of ballistic atom pumps and this is considered from a topological perspective. In a future complementary paper, we will present a thorough examination of ballistic atom pumps using classical, semiclassical, and quantum theories. In earlier work, we have studied three other models of chaotic transport: escape of excited electrons from atoms in strong electric and magnetic fields, escape of light or sound from a vase-shaped enclosure, and escape of points from a region of the phase plane in an abstract two-dimensional map [37-49]. In all of these cases, a classical description of the system leads to a homoclinic tangle, the interior of which we call a "complex," and the trajectories begin on some line in the tangle. When we plot the escape time (the number of iterates of the map required for escape from the complex) versus initial position on that line, we find that the graph has a fractal structure analogous to a Cantor set. At each iterate, some segments of the line of initial conditions escape from the complex. In addition, an infinite sequence of such escape segments, called an epistrophe, converges upon each endpoint of every escape segment.

We developed a topological method (homotopic lobe dynamics) for describing the structure of these escape-time graphs. From computations of early iterates of the map, we extract essential topological information, express that information as a set of symbols, and construct an algebraic map on those symbols that describes the topological structure of forward iterates of the line of initial conditions. That symbolic dynamics predicts a minimal topologically required set of escape segments that must occur in all future iterates.

The purpose of this paper is to move this topological theory forward with a different type of example. A trivial difference is that previously we were considering two-dimensional systems, while here we are considering a one-dimensional system that is periodic in time. A significant difference is that most previous work involved homoclinic tangles, while the present work involves a heteroclinic tangle. (A different heteroclinic tangle, and nested tangles, were also studied in Refs. [50,51].) As a result, the algebra is more complex. The most important difference is that we are here considering a full scattering problem, in which particles come in from infinite distance and eventually recede to infinite distance. (Our earlier studies of escape could be regarded as "half-scattering" problems.) The full scattering problem brings in several new elements to the theory that were not present in the previous papers.

Generalizing and extending homotopic lobe dynamics (HLD) for use with full scattering systems has many purposes. In a ballistic atom pump, the primary goal is to calculate the net flow of particles that approach the barriers from both sides, and HLD allows one to use stable manifolds to place lower bounds on the number of particles which are transmitted or reflected by the barriers for all incoming energies (this is much faster than computing all trajectories). In this paper, HLD is primarily used to explain the fractal structure seen in escape-time graphs. 
In other contexts, HLD can also be used to find a minimal set of closed or periodic orbits $[45,51]$ in order to carry out semiclassical sums, to calculate topological entropy [48-50] (a measure of the complexity of the dynamics), and to partition mixed phase spaces [52].

The structure of the paper is as follows. In Sec. II we select particular parameters for our pump, and we define and compute the escape time graph and display its fractal structure. In Sec. III we sketch the topological theory, with emphasis on the new elements that have to be examined. Then, in Sec. IV, we show the results and compare them with the computations. That is a logical stopping point for readers who do not want to know all the details. The rest of the paper fills in the details of homotopic lobe dynamics for this full scattering problem.

\section{COMPUTATION OF ESCAPE TIMES}

We consider sets of particles approaching the barriers from far away. In the regions far from the barriers, $V(x, t) \approx 0$, so particles effectively travel through them as free particles. As particles approach the oscillating barriers, some of them may not have enough energy to transmit past the first barrier and are directly reflected. Some particles may have enough energy to transmit past both barriers, which we call direct transmission. Other particles may transmit past the first barrier but not have enough energy to transmit past the second barrier. In this case, particles may spend a considerable amount of time in between the barriers, reflecting back and forth from one barrier to the other, before finally reaching the left or right barriers with an appropriate energy and phase to get over the barrier and be transmitted to the right or reflected to the left.

For all graphs in this paper, we choose system parameters $U_{0}=1, \alpha=0.5, \omega=2 \pi / 3, \hat{x}=3, \sigma=1$, and $\phi=\pi / 2$. The separation from the center of one repulsive barrier to the other is $2 \hat{x}=6 \sigma$, so the effect of overlap between the two is negligible. We examine initial conditions such that all particles begin to the left of the barriers with the same initial momentum, $p_{0} \approx 1.50$, but with variable initial position, $-20.9 \lesssim x_{0} \lesssim-16.4$. It is sufficient to analyze a line segment of length $\Delta x_{0}=p_{0} 2 \pi / m \omega$, which is the distance each particle travels during the first cycle of the oscillating barriers. The methods we use naturally extend to any other set of barrier parameters, initial positions, and initial momenta.

We numerically integrate the classical equations of motion for the particles to obtain $x(t)$ and $p(t)$. We monitor $x(t)$ and define a particle's escape time as the amount of time required for the particle to escape the barrier region. Specifically, we say that a particle has escaped to the left if it passes through a line located at $x(t)=-\hat{x}-\gamma$ with negative momentum and that a particle has escaped to the right if it passes through $x(t)=\hat{x}+\gamma$ with positive momentum, where $\gamma=2 \times 10^{-7}$.

Figures 1(a) and 1(b) show continuous escape times (smooth curves) as a function of initial position for particles temporarily trapped between the barriers. Dotted curves (red online) represent particles that escaped to the left, and solid curves (blue online) represent particles that escaped to the right. Also shown are discrete escape times (horizontal lines),

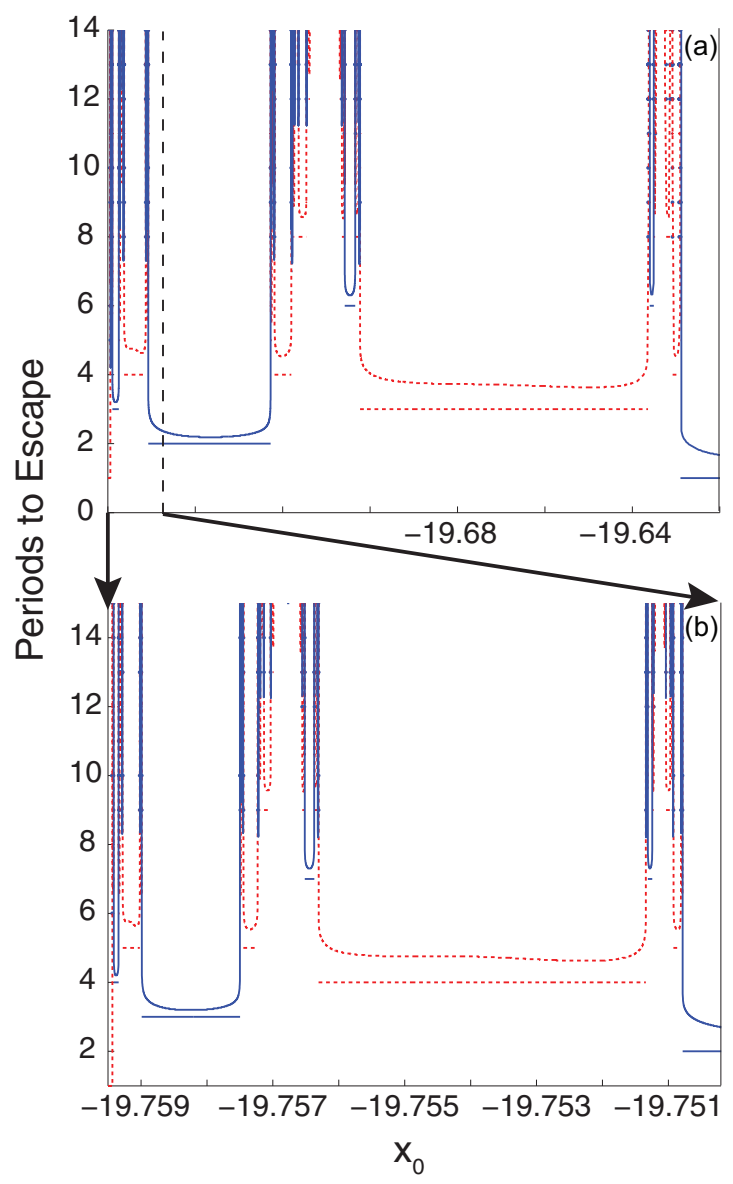

FIG. 1. (Color online) Continuous (smooth curves) and discrete (horizontal lines) escape times as a function of initial position for particles temporarily trapped between the barriers. The dotted (red) curves represent particles that escaped to the left and solid (blue) curves represent particles that escaped to the right. (b) A zoom of the section of (a) between the left edge and the dashed black line, as denoted by the arrows.

which will be discussed later in the paper. The continuous and discrete escape times are plotted relative to a specific time, which is discussed later in the paper.

Fractal behavior is evident in these plots. Figure 1(b) is a zoom of a small region of Fig. 1(a). We see the same structure of icicles but with escape times shifted upward by a time equal to one period of the potential. We see that the same pattern of escape segments occurs on different scales and that it begins at different times in different regions. This type of structure is repeatedly observed when examining even smaller sections of initial position. We also see an additional type of self-similar structure called an "epistrophe." An epistrophe is an infinite sequence of escape segments that converges on the edge of an escape segment $[37,38]$. Every edge of every escape segment has an epistrophe converging upon it.

\section{TOPOLOGICAL ANALYSIS: SKETCH OF THE THEORY}

The purpose of the topological analysis is to provide an interpretation of the structures seen in the escape-time plots. 
The methods are similar to those used in earlier work. In this section we give only a brief sketch of the theory, pointing out the important differences from the systems studied previously, and we show the result in Sec. IV. In later sections we fill in all the details.

First, a surface of section (SOS) must be defined. We strobe the continuous motion once per cycle of the pump, and record $(x, p)$. The SOS has two unstable fixed points, corresponding to particles riding up and down on top of the barriers. The stable and unstable manifolds of these fixed points create a heteroclinic tangle. In much of the earlier work, there was only one fixed point, giving a homoclinic tangle. The present paper is the first application of homotopic lobe dynamics to a heteroclinic tangle for a full scattering system, in which particles come in from an infinite distance. These types of initial conditions, along with the additional fixed point, make this problem somewhat more complex than previous ones.

In previous work, we were studying escape from a complex, sometimes called a half-scattering process. Here we have a full scattering process, in which particles come in from infinite distances. We have to specify an appropriate line of initial conditions, $l_{0}$. This is a line segment of fixed momentum $p_{0}$ having length equal to $\Delta x_{0}=p_{0} T / m$, where $T=2 \pi / \omega$ is the cycle time of the potential. We need to choose the endpoints of this line segment in an appropriate manner. The most convenient choice depends upon the value of the initial momentum, $p_{0}$.

We have to map that line segment forward a sufficient number of times such that particles have first arrived in the pump. We need to define precisely the meaning of that statement. The resulting curve on the SOS is called $L_{0}$.

We also need to compute the structure of the heteroclinic tangle, which is made up of two stable manifolds and two unstable manifolds of the two unstable fixed points. We need to define certain "fundamental segments" of these manifolds, and we need to map the fundamental segments of the unstable manifolds forward some selected number of times, $J$.

The next step is to define certain holes in the plane, which correspond to regions into which stable and unstable manifolds do not enter. The definition of holes given in earlier work is not the optimal choice for the present work, and we give a modified definition of the holes. Then we define bridges, which are usually segments of unstable manifolds. Here we find that to give a topological description of $L_{0}$, we need to create additional bridges; these are curve segments in the SOS that are not segments of any unstable manifold.

Next, the homotopy class of each bridge is defined and named. The homotopy class of a bridge is the family of all curve segments that is made by smoothly distorting the bridge, with the restrictions that the endpoints do not change, and the distortion does not cause the bridge to pass through a hole. Homotopy classes are defined by how the bridges wind around the holes.

The names or symbols of the homotopy classes are used to create a symbolic dynamics. Evolution of each trajectory induces a mapping of each point in the plane, which therefore induces a mapping of curve segments, which induces a mapping of the homotopy classes of those curve segments, and this is expressed as a mapping or algebra of the symbols.

For our chosen set of system parameters and $l_{0}$, we find 10 homotopy classes. Their symbols and the mapping of those symbols are given by

$$
\begin{aligned}
M\left(u_{n}^{t}\right) & =u_{n+1}^{t} \\
M\left(u_{n}^{b}\right) & =u_{n+1}^{b} \\
M\left(c_{1}^{t}\right) & =c_{2}^{t} \\
M\left(c_{1}^{b}\right) & =c_{2}^{b} \\
M\left(c_{2}^{t}\right) & =f^{t b}\left(u_{0}^{b}\right)^{-1}\left(f^{t b}\right)^{-1} \\
M\left(c_{2}^{b}\right) & =f^{b t}\left(u_{0}^{t}\right)^{-1}\left(f^{b t}\right)^{-1} \\
M\left(f^{t b}\right) & =f^{t b} u_{0}^{b} c_{1}^{b} \\
M\left(f^{b t}\right) & =f^{b t} u_{0}^{t} c_{2}^{t} \\
M\left(S_{L L}\right) & =S_{L L} \\
M\left(S_{L T}\right) & =S_{L T} c_{1}^{t} u_{0}^{t} .
\end{aligned}
$$

Next we must ascertain the topological structure of $L_{0}$ relative to the holes in the plane, and express it as a product of those symbols. In our case we find

$$
L_{0}=S_{L L} S_{L T}\left(c_{1}^{t}\right)^{-1}\left(S_{L T}\right)^{-1}
$$

Now the homotopic lobe dynamics is complete. We construct an algebraic representation for each iterate of $L_{0}$, denoted $L_{n}$, by mapping each symbol in Eq. (4) using the symbolic representation given by Eq. (3). $L_{n}$ is obtained by mapping each symbol in $L_{0}$ forward $n$ times. The first few iterates are as follows:

$$
\begin{gathered}
L_{0}=S_{L L} S_{L T}\left(c_{1}^{t}\right)^{-1}\left(S_{L T}\right)^{-1} \\
L_{1}=S_{L L} S_{L T} c_{1}^{t} u_{0}^{t}\left(c_{2}^{t}\right)^{-1}\left(u_{0}^{t}\right)^{-1}\left(c_{1}^{t}\right)^{-1}\left(S_{L T}\right)^{-1} \\
L_{2}=S_{L L} S_{L T} c_{1}^{t} u_{0}^{t} c_{2}^{t} u_{1}^{t} f^{t b} u_{0}^{b}\left(f^{t b}\right)^{-1}\left(u_{1}^{t}\right)^{-1} \\
\times\left(c_{2}^{t}\right)^{-1}\left(u_{0}^{t}\right)^{-1}\left(c_{1}^{t}\right)^{-1}\left(S_{L T}\right)^{-1} \\
L_{3}=S_{L L} S_{L T} c_{1}^{t} u_{0}^{t} c_{2}^{t} u_{1}^{t} f^{t b}\left(u_{0}^{b}\right)^{-1}\left(f^{t b}\right)^{-1} \\
\times u_{2}^{t} f^{t b} u_{0}^{b} c_{1}^{b} u_{1}^{b}\left(c_{1}^{b}\right)^{-1}\left(u_{0}^{b}\right)^{-1}\left(f^{t b}\right)^{-1} \\
\times\left(u_{2}^{t}\right)^{-1} f^{t b} u_{0}^{b}\left(f^{t b}\right)^{-1}\left(u_{1}^{t}\right)^{-1} \\
\times\left(c_{2}^{t}\right)^{-1}\left(u_{0}^{t}\right)^{-1}\left(c_{1}^{t}\right)^{-1}\left(S_{L T}\right)^{-1}
\end{gathered}
$$

The formula for $L_{n}$ tells us the topologically forced structure of the escape-time plot. At the $n^{\text {th }}$ iterate, every instance of $u_{0}^{b}$ or $u_{0}^{t}$ (and their inverses) describes a segment of $L_{n}$ that escapes the complex at the $n^{\text {th }}$ iterate toward the left or right, respectively, and which will not return to the barrier region. These escape segments are forced to occur as a consequence of the topological structure of $L_{0}$ in relation to the holes punctured in the plane. 


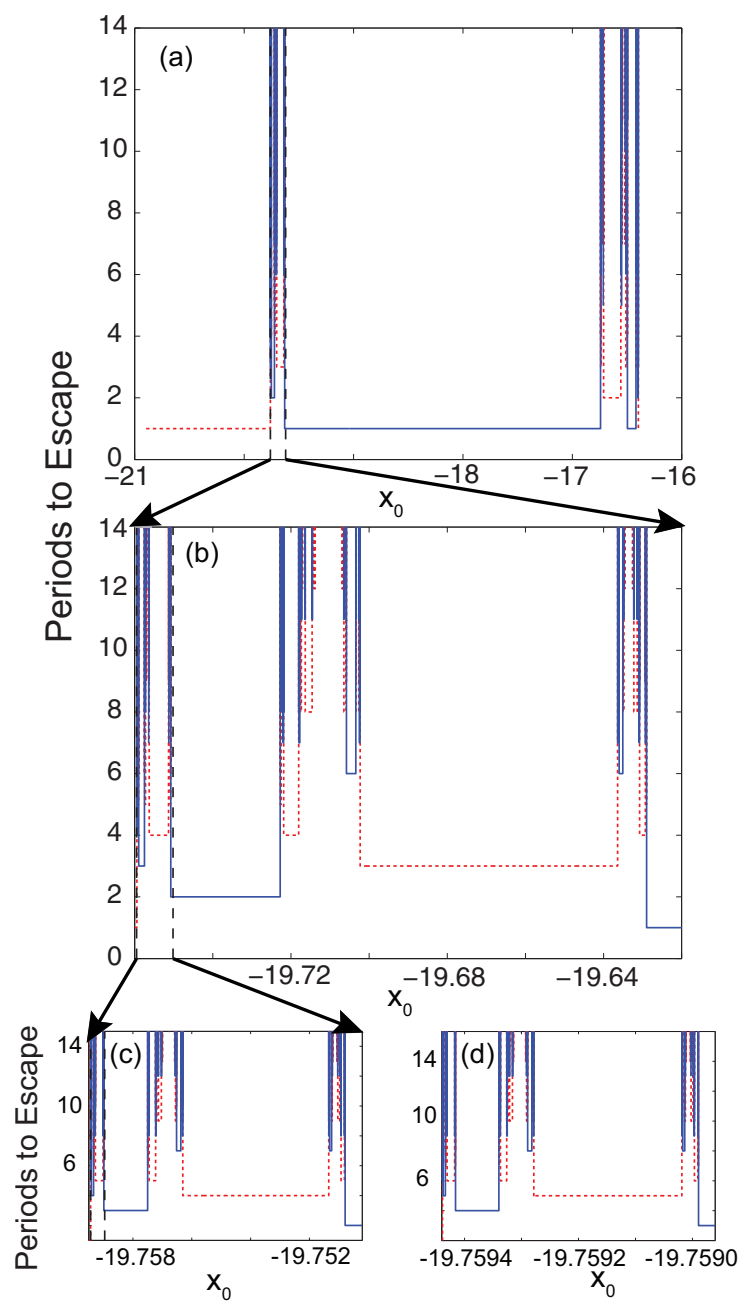

FIG. 2. (Color online) Computed escape segments as a function of position along the initial-condition line. Solid (blue) segments represent transmission, and dotted (red) segments represent reflection. (b) Enlarged region of (a). This segment of $L_{0}$ enters the complex, and some segments are eventually reflected, while others are eventually transmitted. (c) Enlarged region of (b). (d) Enlarged region of (c), from far-left edge of (c) to the dashed vertical black line.

\section{COMPARISON OF TOPOLOGICALLY PREDICTED AND COMPUTED ESCAPE SEGMENTS}

We now compare topologically predicted escape segments with those seen computationally and take note of those that are not predicted by the topology. Figure 2 shows the number of iterates to escape for a directly computed $L_{0}$. To construct this graph, we numerically iterate a high density of points making up $L_{0}$ and calculate the number of iterates at which each point escapes the complex. Dotted (red online) horizontal segments represent reflection (i.e., escape to the left of the barriers), and solid (blue online) horizontal segments represent transmission (i.e., escape to the right of the barriers). In Fig. 2(a), the dotted (red online) segment toward the left of the graph at the first iterate is the segment of $L_{0}$ homotopic to the bridge $S_{L L}$ (see Fig. 13); the solid (blue online) segment in the middle at this iterate is a portion of $S_{L T}$. Neither of these segments ever enters the complex. Their escape at the first iterate (rather than at the zeroth iterate) is a convention chosen to agree with our algebraic method.

Subsequent escape segments of $L_{0}$ have a very complicated structure. Figure 2(b) shows the section of the initial-condition line lying between the two segments that escape at the first iterate in Fig. 2(a). In Fig. 2(b), the dotted (red online) escape segment barely visible at the leftmost edge is the long reflected segment that escapes at the first iterate in Fig. 2(a); the solid (blue online) segment at the rightmost edge is the long transmitted segment that escapes at the first iterate in Fig. 2(a). The section of $L_{0}$ shown in Fig. 2(b) is one of two sections that enters the complex. We see that new escape segments occur at every iterate and that there are many reflected and transmitted segments in this region.

Figures 2(b)-2(d) show a self-similar structure at all levels of resolution. Figure 2(c) shows a small segment of $L_{0}$ seen in Fig. 2(b), and Fig. 2(d) shows a small segment of $L_{0}$ seen in Fig. 2(c). Figures 2(c) and 2(d) show that similar structures seen in Fig. 2(b) repeatedly occur in smaller sections of $L_{0}$ and begin at different iterates. Epistrophes are also present for every edge of every escape segment. The self-similar grouping of escape segments, and their associated epistrophes, are predicted by the algebra.

Let us compare predicted escape segments with numerically computed trajectories. We will look at the specific case of the third iterate. Our algebraic representation for $L_{3}$ is given by Eq. (8). Recall that each instance of $u_{0}^{b}$ (or its inverse) represents a reflected segment (R), and each instance of $u_{0}^{t}$ (or its inverse) represents a transmitted segment (T). Going from left to right in Eq. (8), and counting instances of $u_{0}^{b}$ and $u_{0}^{t}$ (or their inverses), we see that the symbolic representation predicts a total of six escape segments at this iterate, and that their sequence should be TRRRRT. Figure 3 shows the escape segments at the third iterate for directly computed trajectories. Reflected segments are shown by thin gray curves (red online), and transmitted segments are shown by thick gray curves (blue online). In Fig. 3, we see a total of six escape segments at the third iterate, and we see the same sequence of transmitted and reflected segments that were predicted by the algebraic method.

This agreement holds for all iterates in the following sense: A topologically predicted segment must appear in the computation. Sometimes these predicted segments are tiny, and they may be difficult to find in the computation, but they are always present. On the other hand, computation might show additional escape segments that are not predicted topologically

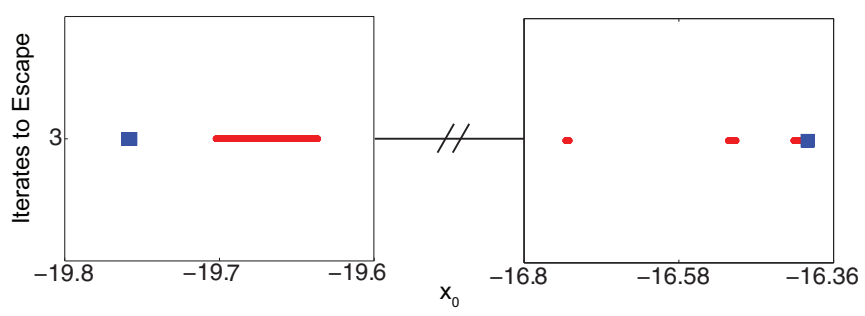

FIG. 3. (Color online) Escape segments seen at the third iterate for directly computed trajectories. Reflected segments are shown by thin gray curves (red), and transmitted segments are shown in thick gray (blue). There are a total of six new escape segments at this iterate, and both the number and the relative order of transmitted and reflected segments are predicted by the algebraic method. 
TABLE I. Escape segments predicted by the level of initial topological information used in the example, and the number of escape segments seen in directly computed trajectories.

\begin{tabular}{lcc}
\hline \hline Iterate & Predicted & Computed \\
\hline 1 & 2 & 2 \\
2 & 3 & 3 \\
3 & 6 & 6 \\
4 & 10 & 10 \\
5 & 16 & 16 \\
6 & 28 & 28 \\
7 & 52 & 56 \\
\hline \hline
\end{tabular}

for the chosen value of $J$. In particular, at high iterates, escape segments that are not forced by the initial topological structure start to emerge. Table I shows the number of escape segments predicted by using $J=3$ and the number of escape segments seen in direct computation of trajectories. For the first six iterates, all computed escape segments are predicted by the algebraic method. At the seventh iterate, however, there are four escape segments seen in computation of trajectories that are not predicted by the algebraic method. This discrepancy is caused by our choice of using only a small anount of initial topological information (three iterates of the fundamental segments of the unstable manifolds). Agreement between the two methods can be extended to higher iterates by using more initial topological information as the basis for the symbolic representation $[47,48]$.

\section{HOMOTOPIC LOBE DYNAMICS FOR A FULL SCATTERING PROBLEM WITH A HETEROCLINIC TANGLE}

In Sec. III, we sketched the topological theory, mentioning the elements that differ from past work. That section should have raised a lot of questions in the reader's mind. In this section, all of those questions are answered. We give all necessary details for carrying out the topological analysis on this type of system. A table of notation is included as Supplemental Material (Ref. [53]) in the online version of this paper, and we strongly encourage the reader to keep a copy of that table handy.

\section{A. Heteroclinic tangle}

We perform our topological analysis on a surface of section for this system. This surface of section is obtained by strobing trajectories at the same phase during each cycle of the potential. We choose our strobe time as $t=3 \pi / 4 \omega$ in each cycle. The surface of section is a continuous map of the $(x, p)$ plane. There are two unstable fixed points, $\mathbf{z}_{x}^{L}=(-\hat{x}, 0)$ and $\mathbf{z}_{x}^{R}=$ $(\hat{x}, 0)$, on the surface of section, each of which corresponds to a particle riding up and down on the top of a barrier. The stable and unstable manifolds of these fixed points are the most important elements of the theory; they define a "complex" or "resonance zone" into which approaching particles can be temporarily captured and from which they later escape. In addition, depending on the parameters of the system, there could be stable periodic orbits of any period which always remain inside the complex.

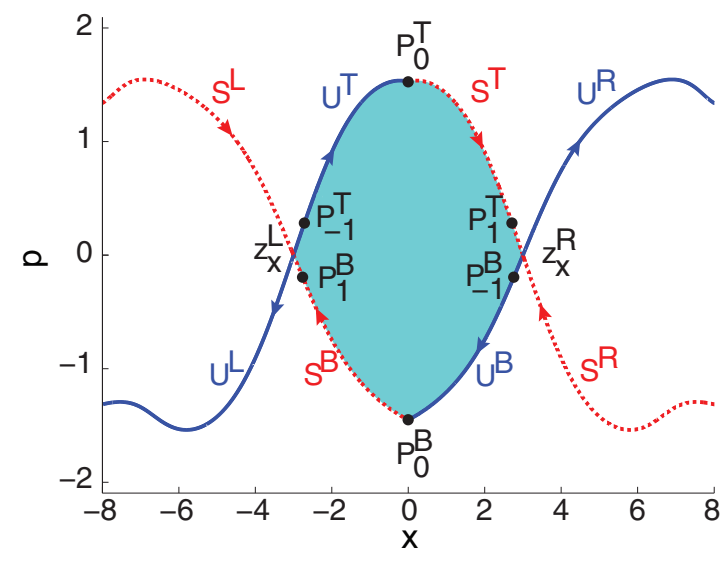

FIG. 4. (Color online) Surface of section showing portions of the four stable [dotted (red) curves] and four unstable [solid (blue) curves] manifolds for two unstable points of the system, $\mathbf{z}_{x}^{L}$ and $\mathbf{z}_{x}^{R}$, for a chosen set of parameters. $\mathbf{z}_{x}^{L}$ is the unstable point at the top of the left barrier, and $\mathbf{z}_{x}^{R}$ is the unstable point at the top of the right barrier. Unstable manifolds asymptotically approach an unstable point as $t \rightarrow-\infty$, and stable manifolds asymptotically approach an unstable point as $t \rightarrow+\infty$. The shaded area (blue) is called the "complex" or "resonance zone" and is discussed in the text.

Figure 4 shows segments of the eight stable and unstable manifolds for this system for a selected set of parameters. Together, these manifolds form a heteroclinic tangle. Different choices of parameters change the details of these manifolds but leave essential topological properties unchanged. This type of tangle is sometimes referred to as a ternary horseshoe [54]. The stable manifold $S^{L}$ comes from the upper-left quadrant of the surface of section and approaches the left-hand fixed point $\mathbf{z}_{x}^{L}$, and the unstable manifold $U^{L}$ goes from that point into the lower-left quadrant. Likewise, the stable manifold $S^{R}$ comes from the lower-right quadrant and approaches the fixed point $\mathbf{z}_{x}^{R}$, and the unstable manifold $U^{R}$ goes from that point into the upper-right quadrant.

Another unstable manifold leaves $\mathbf{z}_{x}^{L}$ going toward the upper right. We call this manifold $U^{T}$, and a segment of it, called $\mathbb{U}^{T}$, corresponds to the left half of the top boundary of the complex. Similarly, a stable manifold $S^{B}$ approaches $\mathbf{z}_{x}^{L}$ from the lower right, and a segment of it, called $\mathbb{S}^{B}$, makes the left half of the lower boundary of the complex. Likewise, $U^{B}$ (and its segment $\mathbb{U}^{B}$ ) and $S^{T}$ (and its segment $\mathbb{S}^{T}$ ) connect with the right-hand fixed point $\mathbf{z}_{x}^{R}$ and also define the right halves of the lower and upper boundaries of the complex.

$U^{T}$ and $S^{T}$ are reflections of each other about the line $x=0$, and they intersect at a heteroclinic point $P_{0}^{T}$ having $(x=0, p>0)$, which we call the top primary intersection point. The segments $\mathbb{U}^{T}$ and $\mathbb{S}^{T}$ go from their respective fixed points to $P_{0}^{T}$. Similarly, $U^{B}$ and $S^{B}$ are reflections of each other through $x=0$, and they intersect at a heteroclinic point which we call the bottom primary intersection point, $P_{0}^{B}$, having $(x=0, p<0) . \mathbb{U}^{B}$ and $\mathbb{S}^{B}$ are segments of these manifolds going from their respective fixed points to $P_{0}^{B}$.

The segments $\mathbb{U}^{T}, \mathbb{S}^{T}, \mathbb{U}^{B}$, and $\mathbb{S}^{B}$ define the outer boundaries of the shaded region (blue online) that we call the complex in Fig. 4. No stable manifold can intersect itself 
or any other stable manifold, and no unstable manifold can intersect itself or any other unstable manifold.

In Fig. 4, when the top primary intersection point, $P_{0}^{T}$, is mapped forward one time, it maps to the point on $\mathbb{S}^{T}$ called $P_{1}^{T}$. The segment $S^{T}\left[P_{0}^{T}, P_{1}^{T}\right)$ is called the fundamental segment, $S_{0}^{T}$, of $S^{T}$. Note that it is not the entire segment, $\mathbb{S}^{T}$, which extends from $P_{0}^{T}$ to $\mathbf{z}_{x}^{R}$. When $P_{0}^{T}$ is mapped backward one time, it maps to the point $P_{-1}^{T}$ on $\mathbb{U}^{T}$, and the fundamental segment of $U^{T}, U_{0}^{T}$, is the segment $U^{T}\left[P_{-1}, P_{0}\right)$. Similarly, the bottom primary intersection point $P_{0}^{B}$ maps forward to $P_{1}^{B}$ on $\mathbb{S}^{B}$ and backward to $P_{-1}^{B}$ on $\mathbb{U}^{B}$. The fundamental segment, $S_{0}^{B}$, of $S^{B}$ is the segment $S^{B}\left[P_{0}^{B}, P_{1}^{B}\right)$, and the fundamental segment of $U^{B}, U_{0}^{B}$, is the segment $U^{B}\left[P_{-1}^{B}, P_{0}^{B}\right)$.

The forward mapping of $U_{0}^{T}$ can be expressed as $M\left(U_{0}^{T}=\right.$ $\left.U^{T}\left[P_{-1}^{T}, P_{0}^{T}\right)\right)=U_{1}^{T}=U^{T}\left[P_{0}^{T}, P_{1}^{T}\right)$, where $M$ is the symbol for the mapping. Generally, for all $n, M^{n}\left(U_{0}^{T}\right)=U_{n}^{T}$ and $M^{n}\left(U_{0}^{B}\right)=U_{n}^{B}$. Each $U_{n}^{T}$ is the segment $U^{T}\left[P_{n-1}^{T}, P_{n}^{T}\right)$, and each $U_{n}^{B}$ is the segment $U^{B}\left[P_{n-1}^{B}, P_{n}^{B}\right)$. Similarly, for all $n$, each $S_{n}^{B}$ is the segment $S^{B}\left[P_{n}^{B}, P_{n+1}^{B}\right)$ and each $S_{n}^{T}$ is the segment $S_{n}^{T}\left[P_{n}^{T}, P_{n+1}^{T}\right)$.

As $U_{0}^{T}$ and $U_{0}^{B}$ are mapped forward, and $S_{0}^{T}$ and $S_{0}^{B}$ are mapped backward, stable and unstable manifolds intersect each other an infinite number of times. Intersections occur at the collection of points $P^{B}=\left(P_{-\infty}^{B}, \ldots, P_{0}^{B}, \ldots, P_{\infty}^{B}\right)$ and at the collection of points $P^{T}=\left(P_{-\infty}^{T}, \ldots, P_{0}^{T}, \ldots, P_{\infty}^{T}\right)$ on the top and bottom boundaries of the complex, respectively. Both $P^{T}$ and $P^{B}$ are heteroclinic orbits, and these points are the endpoints for $U_{n}^{T}, U_{n}^{B}, S_{n}^{T}$, and $S_{n}^{B}$ segments. In addition, for all $n>0, U_{n}^{T}$ intersects $\mathbb{S}^{T}$ exactly once between $U^{T}\left[P_{n-1}^{T}, P_{n}^{T}\right)$, at a point we call $Q_{n}^{T}$. These points can be mapped forward and backward and are another heteroclinic orbit, which we call $Q^{T}=\left(Q_{-\infty}^{T}, \ldots, Q_{0}^{T}, \ldots, Q_{\infty}^{T}\right)$. For $n<0$, the points $Q_{n}^{T}$ lie on $\mathbb{U}^{T}$. Similarly, for all $n>0, U_{n}^{B}$ intersects $\mathbb{S}^{B}$ exactly once between $U^{B}\left[P_{n-1}^{B}, P_{n}^{B}\right)$, at a point we call $Q_{n}^{B}$. These points can be mapped forward and backward and are another heteroclinic orbit, which we call $Q^{B}=\left(Q_{-\infty}^{B}, \ldots, Q_{0}^{B}, \ldots, Q_{\infty}^{B}\right)$. For $n<0$, the points $Q_{n}^{B}$ lie on $\mathbb{U}^{B}$.

As can be seen in Fig. 5, segments $U_{n}^{T}$ and $S_{n-1}^{T}$ lie between $P_{n-1}^{T}$ and $P_{n}^{T}$ and intersect at $Q_{n}^{T}$. Similarly, each $U_{n}^{B}$ and $S_{n-1}^{B}$ lie between $P_{n-1}^{B}$ and $P_{n}^{B}$ and intersect at $Q_{n}^{B}$. These segments form the boundaries of lobes. For example, the segments of $U_{1}^{T}$ and $S_{0}^{T}$ between $P_{0}^{T}$ and $Q_{1}^{T}$ bound a lobe labeled $C_{1}^{T}$, and the segments of $U_{1}^{T}$ and $S_{0}^{T}$ between $Q_{1}^{T}$ and $P_{1}^{T}$ bound another lobe called $E_{0}^{T}$. The points $P_{0}^{T}$ and $Q_{1}^{T}$ map forward to points $P_{1}^{T}$ and $Q_{2}^{T}$, respectively, and the stable and unstable manifold segments connecting them bound the lobe $C_{2}^{T}$, which is the forward mapping of lobe $C_{1}^{T}$. Generally, the two lobes bounded by $U_{n}^{B, T}$ and $S_{n-1}^{B, T}$ map forward to two lobes bounded by $U_{n+1}^{B, T}$ and $S_{n}^{B, T}$ and map backward to two lobes bounded by $U_{n-1}^{B, T}$ and $S_{n}^{B, T}$.

The lobes are crucial for understanding the transport process. Since this system is Hamiltonian, the mapping is area preserving, meaning that all forward and backward iterates of a given lobe have the same area. When the endpoints of manifold segments bounding a lobe are on $\mathbb{S}^{T}$ (or $\mathbb{S}^{B}$ ), their forward iterates get closer together along $\mathbb{S}^{T}$ (or $\mathbb{S}^{B}$ ) as $n \rightarrow \infty$. Consequently, the lobe's forward iterates must stretch in order to preserve the same area. Similarly, when the
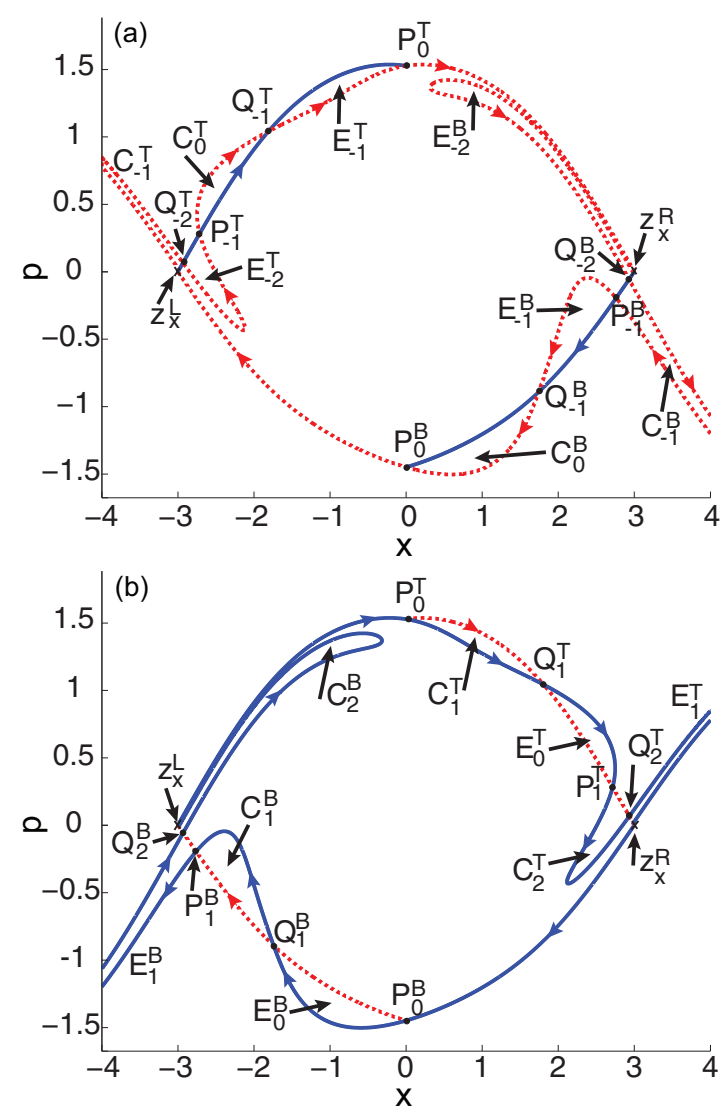

FIG. 5. (Color online) (a) $\mathbb{S}^{T}, \mathbb{S}^{B}$, and the first two back iterates of $S_{0}^{T}$ and $S_{0}^{B}$ (dotted red curves), along with $\mathbb{U}^{T}$ and $\mathbb{U}^{B}$ [solid (blue) curves]. Each back iterate of the fundamental stable segments forms two lobes with either $\mathbb{U}^{T}$ or $\mathbb{U}^{B}$. (b) $\mathbb{U}^{T}, \mathbb{U}^{B}$, and the first two forward iterates of $U_{0}^{T}$ and $U_{0}^{B}$ [solid (blue) curves], along with $\mathbb{S}^{T}$ and $\mathbb{S}^{B}$ [dotted (red) curves]. Each forward iterate of the unstable fundamental segments forms two lobes with either $\mathbb{S}^{T}$ or $\mathbb{S}^{B}$. The lobes $C_{0}^{T}$ and $C_{0}^{B}$ in (a) map from outside the complex to the lobes $C_{1}^{T}$ and $C_{0}^{B}$, respectively, in (b), which are inside the complex. The lobes $E_{-1}^{T}$ and $E_{-1}^{B}$ in (a) map forward to the lobes $E_{0}^{T}$ and $E_{0}^{B}$, respectively, in (b), which are outside the complex.

endpoints of manifold segments bounding a lobe are on $\mathbb{U}^{T}$ (or $\mathbb{U}^{B}$ ), their backward iterates get closer together along $\mathbb{U}^{T}$ (or $\mathbb{U}^{B}$ ) as $n \rightarrow-\infty$, and again the lobes must stretch in order to preserve the same area.

In Fig. 5, we can see that some lobes are inside the complex and others are outside it. Two lobes, labeled $C_{0}^{B}$ and $C_{0}^{T}$, lie outside the complex, but their forward mappings, labeled $C_{1}^{B}$ and $C_{1}^{T}$, respectively, lie inside the complex. The areas inside $C_{0}^{B}$ and $C_{1}^{B}$ are equal (as are the areas inside $C_{0}^{T}$ and $C_{1}^{T}$ ). This means that any particle in the phase plane that is inside the lobe $C_{0}^{T}$ at any strobe time will be inside $C_{1}^{T}$ one cycle later, i.e., the particle will be "captured" or transported from outside the complex to within it. At the next cycle, the particle will be in $C_{2}^{T}$, and so on. The same process occurs for the lobes labeled $C_{0}^{B}, C_{1}^{B}$, and $C_{2}^{B}$.

Another pair of important lobes are labeled $E_{-1}^{T}$ and $E_{-1}^{B}$. They are inside the complex but their forward iterates, labeled $E_{0}^{T}$ and $E_{0}^{B}$, respectively, lie outside the complex. Any particle in the phase plane inside either $E_{-1}^{T}$ or $E_{-1}^{B}$ at any strobe time 
will be inside $E_{0}^{T}$ or $E_{0}^{B}$, respectively, one cycle later, and will have "escaped," i.e., been transported from within the complex to outside of it. More generally, a particle inside a lobe $E_{-n}^{T}$ or $E_{-n}^{B}$ will escape the complex $n$ cycles later. Particles inside $E_{-n}^{T}$ will eventually escape to the right of both barriers, and particles inside $E_{-n}^{B}$ will escape to the left of the barriers.

When Figs. 5(a) and 5(b) are plotted together, one can see that capture and escape lobes within the complex intersect each other in very complicated fashion [see Fig. 7(a)]. However, they allow us to understand the transport process in the phase plane. We will use them later to develop a method for understanding the escape-time plots seen earlier.

\section{B. Initial conditions}

In the past, because we were studying escape, lines of initial conditions were typically chosen to lie in the complex. However, since we now wish to study a scattering process, having particles approaching from $x= \pm \infty$ with a constant momentum $p_{0}$, we take our line of initial conditions $l_{0}$ to be a horizontal line segment in phase space far away from the barriers, where the potential is effectively zero. We also define this line segment such that its length is $\Delta x_{0}=p_{0} T / \mathrm{m}$, where $T=2 \pi / \omega$ is the cycle time of the barriers and such that it has endpoints on a stable manifold. This allows us to describe the topological structure of the evolution of initial-condition lines in terms of the evolution of bridges, which are segments of the unstable manifolds.

Let $\mathscr{L}$ represent $l_{0}$ and all its iterates. The curve $\mathscr{L}$ cannot intersect itself. If it intersected itself on a particular iterate, it would intersect itself at every iterate. However, by construction, $l_{0}$ does not intersect itself. If one iterate in $\mathscr{L}$ intersected another iterate in $\mathscr{L}$, then mapping that intersection backward many times, there would have to be a corresponding intersection on $l_{0}$. However, at early times, the line of initial conditions represents particles of fixed momentum moving toward the barriers. No future iterate can intersect that line, because any particle reflected from the barriers (back toward the initial-condition line) will be traveling in the opposite direction of the initial-condition line and will thus be in a different region of the phase plane.

While $l_{0}$ represents particles approaching the barriers from $x= \pm \infty$, we will base our topological analysis on a selected forward-iterate of $l_{0}$, called $L_{0}$, that has one of the following properties. If particles approach from the left,

Type I: Its endpoints both lie on $\mathbb{S}^{T}$.

Type II: Its endpoints both lie on $S^{L}$, and it intersects $\mathbb{S}^{T}$.

If particles approach from the right,

Type III: Its endpoints both lie on $\mathbb{S}^{B}$.

Type IV: Its endpoints both lie on $S^{R}$, and it intersects $\mathbb{S}^{B}$.

$L_{0}$ should be chosen at the earliest iterate of $l_{0}$ satisfying one of these conditions. In order for the endpoints of $L_{0}$ to lie on a stable manifold, the endpoints of its preiterate $l_{0}$ must also lie on the same stable manifold. Therefore, we must examine $S^{T}, S^{B}, S^{L}$, and $S^{R}$ at many backward iterates, far from the barriers, when constructing $l_{0}$.

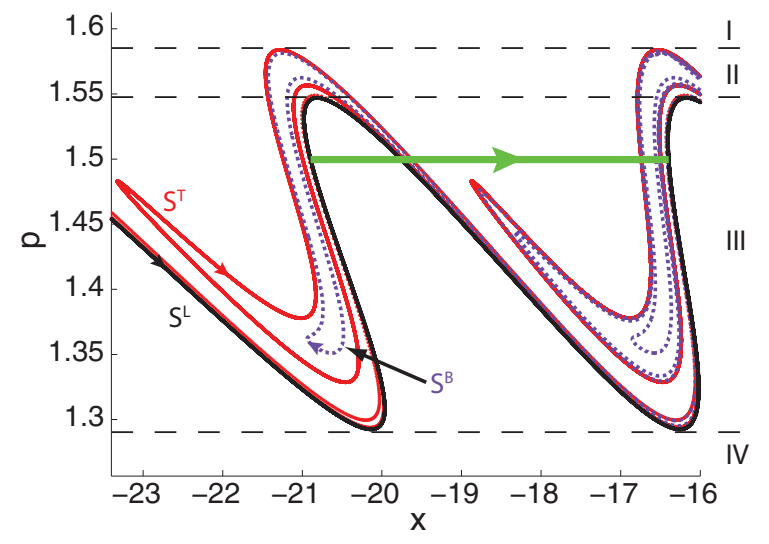

FIG. 6. (Color online) Backward iterates of $S^{T}$ [uppermost solid (red) curve], $S^{B}$ [dotted (purple) curve], and $S^{L}$ [lowermost (black) curve] in a region far to the left of the barriers, where the potential is effectively zero, for a chosen set of parameters. Initial-condition lines in the regions labeled I-IV scatter from the barriers with qualitatively different behavior, which is discussed in the text. Also shown is an example $l_{0}$ [horizontal (green) segment], constructed with the rules discussed in the text, which has an initial momentum corresponding to Region III.

In the rest of this paper, we consider only particles approaching from the left, though the topological analysis is analogous for particles approaching from the right. Figure 6 shows $S^{L}$ [lowermost solid (black online) curve], $S^{T}$ [uppermost solid (red online) curve], and $S^{B}$ [dotted (purple online) curve] at many backward iterates in a region far to the left of both barriers. $S^{R}$ cannot enter this region (see Fig. 4). The qualitative properties of the scattering depend upon the initial momentum and, specifically, on whether $l_{0}$ lies in Region I, II, III, or IV. These regions are determined according to maxima and minima of $S^{T}$ and $S^{L}$.

If the initial momentum is large, so $l_{0}$ is in Region I (above the max of $S^{T}$ in this region), $\mathscr{L}$ will never intersect a stable manifold. Since it begins above $S^{T}$, it will be directly transmitted past both barriers without entering the complex.

If the initial momentum is such that $l_{0}$ is in Region II (below the max of $S^{T}$ and above the max of $S^{L}$ in this region), a portion of $\mathscr{L}$ will be directly transmitted past both barriers without entering the complex, and another portion of $\mathscr{L}$ will enter the complex. The segments that lie above $S^{T}$ in this region will be directly transmitted, and those that lie below $S^{T}$ will enter the complex. Portions of segments entering the complex can be either reflected or transmitted. The endpoints of $l_{0}$ should be placed on the uppermost segments of $S^{T}$ in this region, so it will evolve into a Type I $L_{0}$.

For initial momentum such that $l_{0}$ lies in Region III (between the min and max of $S^{L}$ in this region), portions of $\mathscr{L}$ can be directly transmitted or directly reflected without entering the complex, and portions can enter the complex. The endpoints of $l_{0}$ should be placed on $S^{L}$, so it will evolve into a Type II $L_{0}$. An example $l_{0}$ [horizontal (green online) curve] is shown for $p_{0} \approx 1.50$ in Fig. 6 , with its endpoints on $S^{L}$. If the initial momentum is small, so $l_{0}$ lies in Region IV, $\mathscr{L}$ will never intersect a stable manifold, cannot enter the complex, and will be directly reflected. 
For initial momenta in Regions II and III, intersections between $l_{0}$ and any stable manifold will map to an unstable point. Our use of initial-condition lines with width $\Delta x_{0}=$ $p_{0} 2 \pi / m \omega$ ensures that both endpoints of $l_{0}$ lie on the same stable manifold. Similar analysis for $l_{0}$ lines representing particles approaching from the right would allow us to construct $L_{0}$ of Types III and IV.

\section{Homotopic lobe dynamics}

Stable and unstable manifolds forming a heteroclinic tangle intersect each other an infinite number of times. Since stable manifolds cannot intersect themselves or any other stable manifold, and unstable manifolds cannot intersect themselves or any other unstable manifold, segments of stable and unstable manifolds connecting a pair of intersection points can create domains which no stable or unstable manifold can enter. These domains are part of the structure of the heteroclinic tangle and are a main component of the topological analysis.

Another important aspect is that, as initial-condition lines evolve, they follow and approach unstable manifolds in the chaotic regions of the phase plane. For this reason, we topologically analyze the evolution of unstable manifolds, which we then relate to the evolution of initial-condition lines. Rather than analyze the entirety of each unstable manifold at once, we instead analyze how segments of unstable manifolds evolve. These segments are classified according to how they wind around the domains that manifolds cannot enter.

We then develop a symbolic algebra describing how the unstable manifold segments evolve. The initial topological structure of the unstable manifold segments in relation to the manifold-excluding domains forces a minimal topological structure at later times. The algebra predicts this structure and tells us both the minimum number and relative order (along $L_{0}$ ) of new escape segments at each forward iterate. One chooses the amount of topological information (i.e., the length of the stable and unstable manifolds) to use for the basis of analysis. Using more initial information may allow additional structure to be predicted at later times.

\section{Fundamental segments}

To set up the topological theory, we map the fundamental segments $U_{0}^{B}$ and $U_{0}^{T}$ forward $J$ times. As stated earlier, choosing a higher $J$ may provide additional topological information, which in turn may allow additional escape segments to be predicted at later times. The solid dark gray (blue online) curves in Fig. 7(a) show $U_{0}^{T}$ and $U_{0}^{B}$ mapped forward $J=3$ times, and $\mathbb{U}^{T}$ and $\mathbb{U}^{B}$. The dotted curves (red online) show $S_{0}^{T}$ and $S_{0}^{B}$ mapped backward $J=3$ times, along with $\mathbb{S}^{T}$ and $\mathbb{S}^{B}$, for our selected set of parameters. The back-iterates of $S_{0}^{T}$ and $S_{0}^{B}$ shown in Fig. 7(a) are not needed for the topological method but are shown to help the reader visualize the heteroclinic tangle.

\section{J-neighbors}

We must find domains that manifolds cannot enter. Each domain of this type is bounded by a stable manifold segment and an unstable manifold segment that intersect at two points. A pair of intersection points are called neighbors if both
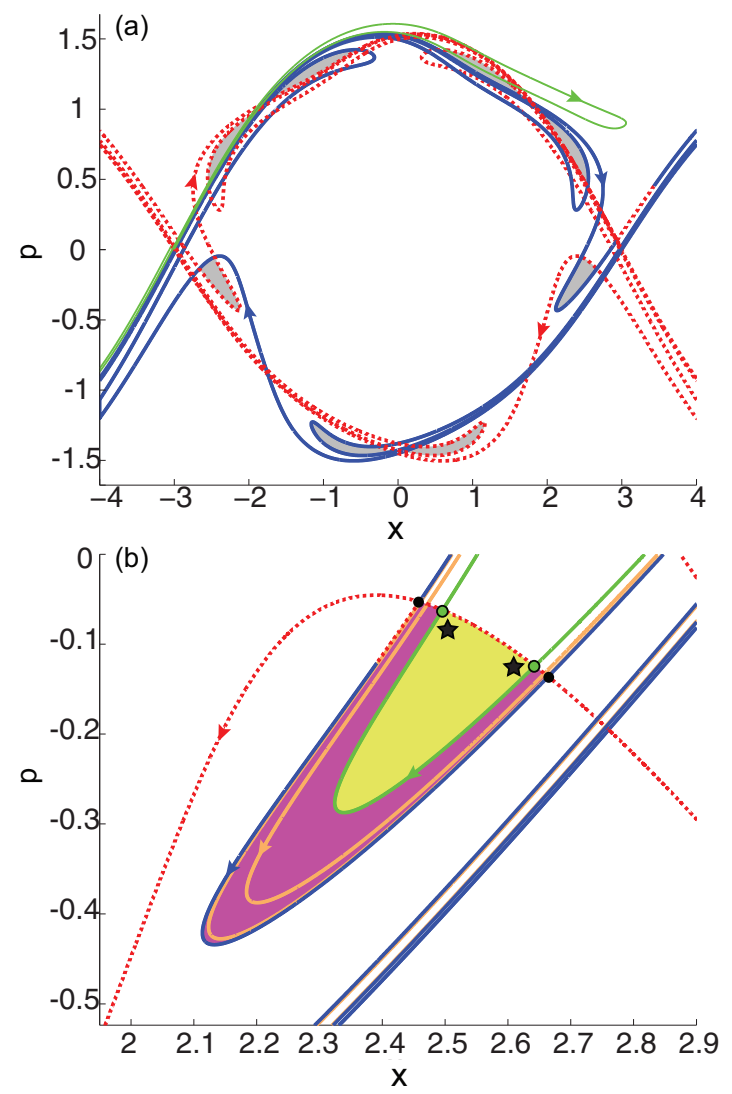

FIG. 7. (Color online) (a) Unstable manifolds [dark gray (blue) solid curves] iterated forward $J=3$ times, and stable manifolds [dotted (red) curves] iterated backwards $J=3$ times. The uppermost solid curve [light gray (green)] is $L_{0}$. (b) Zoom of a domain-hole from (a). The shaded regions (yellow and pink) combined constitute the domain-hole, into which no $S$ or $U$ can enter. In Ref. [49], we shrunk such domain-holes to point-holes such that the point was inside the domain but arbitrarily close to one of the two heteroclinic points on the boundary of the domain (large black dots). In this paper, we use a different choice of point-hole: either of the points marked by a $\star$, which is inside the subhole [light gray (yellow)] and arbitrarily close to one of the $J$-subneighbors [large gray (green) dots].

the stable and unstable manifold segments connecting them contain no other intersections with any stable or unstable manifolds. In practice, it is difficult to determine whether a pair of intersection points are neighbors; they may appear to be neighbors at some iterate $J$, but at some other iterate $J^{\prime}>J$, previously unseen intersections may be revealed.

For this reason, we work with $J$-neighbors, which depend on the number of iterates, $J$, used for the topological analysis. To define them, we must define the transition number $N$ of an intersection $\mathbf{z}$, which is the number of iterates $N$ such that $M^{N+m}(\mathbf{z})$ lies on either $S_{0}^{T}$ or $S_{0}^{B}$, when $M^{m}(\mathbf{z})$ lies on either $U_{0}^{T}$ or $U_{0}^{B}$.

Two intersection points are $J$-neighbors if (i) they both have transition number $N \leqslant J$ and (ii) the stable and unstable manifold segments connecting them contain no other intersection points with transition number $N \leqslant J$.

An efficient method for finding $J$-neighbors for the chosen $J$ is to iterate the fundamental unstable manifold segments 
$U_{0}^{T}$ and $U_{0}^{B}$ forward $J$ times and examine heteroclinic intersections along $S_{0}^{T}$ and $S_{0}^{B}$. Only adjacent pairs of heteroclinic points along $S_{0}^{T}$ and $S_{0}^{B}$ can possibly be $J$-neighbors. Furthermore, any intersection between $M^{k}\left(U_{0}^{T}\right)$ or $M^{k}\left(U_{0}^{B}\right)$ and $S_{0}^{T}$ or $S_{0}^{B}$ has transition number $N=k$. The $J$-neighbors found in this fashion can be mapped forward and backward to find all other pairs of $J$-neighbors for the chosen $J$.

Since the stable and unstable manifold segments connecting a pair of neighbors have no other intersection points, they bound a domain in the phase plane that no manifold can enter. The topological theory describes the evolution of unstable manifolds in relation to these manifold-excluding domains. One may think of $J$-neighbors as pairs of intersection points that appear to be neighbors through the chosen iterate $J$, i.e., the stable and unstable manifold segments connecting them bound a domain for which it appears that no manifold can enter. We use these manifold-excluding domains in the topological theory.

\section{Holes}

To describe the topological structure of curves, we need to punch holes in the plane and specify how the curves go around the holes. In the past, we used two different definitions of holes. In the first paper [38], a hole was a domain of the plane into which iterates of stable and unstable manifolds could not enter. In a later paper [49], we shrunk the holes to carefully selected points within the previously used domains. In this paper we find it best to shrink the domain-holes to a different set of point-holes.

Figure 7 (a) shows $L_{0}, \mathbb{U}^{T}, \mathbb{U}^{B}, \mathbb{S}^{T}, \mathbb{S}^{B}$, the first three forward iterates of $U_{0}^{T}$ and $U_{0}^{B}$, and the first three back-iterates of $S_{0}^{T}$ and $S_{0}^{B}$. Domain-holes are shaded in gray, and no forward or backward iterate of $S^{T}, S^{B}, U^{B}$, or $U^{T}$ enters these domain-holes for the chosen $J$. Figure 7(b) shows a zoom of one domain-hole (shaded region; yellow and pink online) and a portion of $\mathscr{L}$. It can and does enter the domain-hole. The uppermost solid curve (green online) which enters the domain-hole is $L_{1}$, and the two solid curves below it (orange online) which enter the domain-hole are portions of $L_{2}$. Computations have shown that this commonly occurs for appropriately constructed $L_{0}$. In this system, each time a portion of $\mathscr{L}$ enters a domain-hole, it will be closer to the unstable manifold bounding the domain-hole than the last time it entered, because points and curves are mapped forward toward and along unstable manifolds.

Each domain-hole is associated with a pair of $J$-neighbors. The boundary of the domain-hole is made up of a segment of an unstable manifold connecting the $J$-neighbors and a segment of a stable manifold connecting them. In the past, each domain-hole was shrunk to a point and "moved" arbitrarily close to one of the $J$-neighbors associated with it while remaining in the domain of the original domain-hole. Point-holes were then mapped forward and backward to represent domain-holes in the plane. Since we described the evolution of the manifolds (and lines of initial conditions) in terms of how they wound around domain-holes, using a point-hole to represent a domain-hole was sufficient, because a point-hole is within the domain of the domain-hole.

However, for this system, this method results in loss of important information; see Fig. 7(b). If we use a point-hole that is arbitrarily close to one of the $J$-neighbors (large black dots), we see that the segments of $\mathscr{L}$ no longer wind around the pointhole in the same fashion as the unstable manifold segment which they approach. Since we want to describe the evolution of $L_{0}$ in terms of the way unstable manifold segments evolve in relation to point-holes, we must develop a method for selecting a point-hole such that both $\mathscr{L}$ and the unstable manifold it approaches wind around the point-hole in the same fashion.

In the present case we see that $\mathscr{L}$ enters the domain-hole. The uppermost solid curve (green online) which enters the domain-hole in Fig. 7(b) is $L_{1}$. All forward iterates of $L_{1}$ that enter this domain-hole lie in the darker gray (pink online) region, between $L_{1}$ and the unstable manifold segment bounding the domain-hole, and not in the light gray (yellow online) region. This occurs because $\mathscr{L}$ approaches the unstable manifold; $L_{2}$, which passes through the domain-hole twice, is the solid curve (orange online) passing through the darker gray (pink online) portion of the domain-hole. Therefore, we may call the light gray (yellow online) region a subhole. In this paper we define new point-holes by shrinking the light gray (yellow online) subdomain to either one of the points marked $\star$ inside the subdomain but arbitrarily close to one of the intersections of $L_{1}$ with $S^{B}$ [large gray (green online) dots]. $L_{1}$ then goes around that hole, subsequent iterates of $L_{1}$ also go around it, and they must also go around iterates of that hole.

When a subhole is formed, there are two intersections between $L_{n}$ and the stable manifold. Let us call these intersections $J$-subneighbors. The $J$-subneighbors are connected by two curves: a segment of $L_{n}$ and a segment of the stable manifold.

With these ideas, we can use the following framework to determine placement of point-holes. We first compute the $L_{0}$ we wish to examine. We next determine all iterates of $U_{0}^{T}$ and $U_{0}^{B}$ up to some iterate $J$. We then determine all pairs of $J$-neighbors that lie on $S_{0}^{T}$ and $S_{0}^{B}$. We map each pair of $J$-neighbors forward $J-k$ times, and backward $k$ times, where $k$ is the transition number of the $J$-neighbors. We then see if $J$-subneighbors (intersections between $L_{0}$ and stable manifold segments connecting $J$-neighbors) are present. If no $J$-subneighbors are present, we puncture a point-hole in the plane inside the domain-hole along $S_{0}^{T}$ and $S_{0}^{B}$ and arbitrarily close to one of the $J$-neighbors. We then map this point-hole forward $J-k$ times, and backward $k$ times.

If $J$-subneighbors exist between any pair of $J$-neighbors (or their iterates), a subhole is formed. If there is only one pair of $J$-subneighbors between a pair of $J$-neighbors, we puncture a point-hole in the plane within the subhole and arbitrarily close to either $J$-subneighbor. We then map this point-hole forward and backward the appropriate number of times.

Puncturing point-holes in the phase plane that are developed in this fashion allows us to describe the topological evolution of initial-condition lines to the same level of accuracy as previous methods.

\section{Bridges}

Bridges are curves that have endpoints on $\mathbb{S}^{T}$ or $\mathbb{S}^{B}$ but do not otherwise intersect either of them. In past cases, each of these curves was a segment of an unstable manifold that was part of the boundary of a lobe. $S^{L}$ and $S^{T}$ may intersect bridges, but only if the segment intersecting the bridge is not 


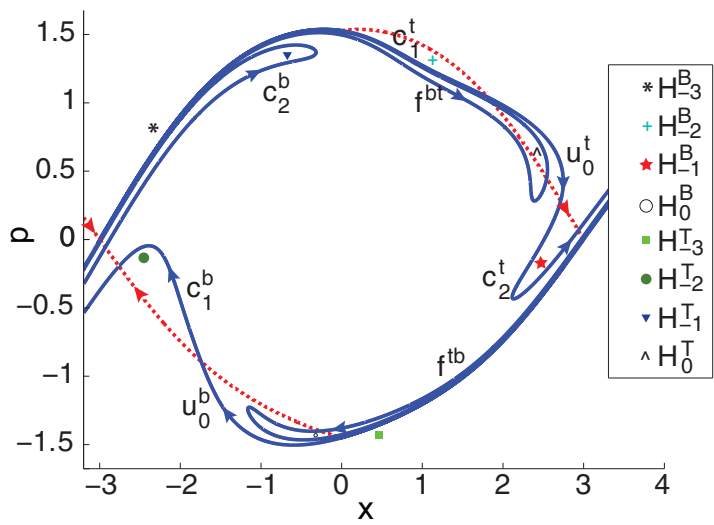

FIG. 8. (Color online) $\mathbb{U}^{T}$ and $\mathbb{U}^{B}, U_{0}^{T}$ and $U_{0}^{B}$, and their first $J=3$ forward iterates [solid (blue) curves], and $\mathbb{S}^{T}, S^{L}$, and $\mathbb{S}^{B}$ [dotted (red) curves] for a given set of parameters. The markers represent point-holes punched in the appropriate places in the phase plane. Bridge classes are constructed according to how these unstable manifold segments wind around the punctured point-holes.

part of $\mathbb{S}^{T}$ or $\mathbb{S}^{B}$. Bridges can begin and end on $\mathbb{S}^{T}$, begin and end on $\mathbb{S}^{B}$, or have one endpoint on $\mathbb{S}^{T}$ and the other on $\mathbb{S}^{B}$. Bridges are directed curves and have the direction of the local unstable manifold. If a curve is not a segment of an unstable manifold, we choose its direction arbitrarily.

Homotopic lobe dynamics describes the topological evolution of bridges, i.e., the way they wind around the punctured point-holes in the plane. After puncturing all point-holes in the plane, we classify each bridge formed from iterating $U_{0}^{T}$ and $U_{0}^{B}$ forward $J$ times according to its topological properties: (i) which stable manifold(s) its endpoints lie on and (ii) which point-hole(s) it surrounds (if any).

Figure 8 shows $U_{0}^{T}$ and $U_{0}^{B}$ and their first $J=3$ forward iterates [solid (blue) curves], along with $\mathbb{S}^{T}, \mathbb{S}^{B}$, and $S^{L}$ [dotted (red) curves] and the punctured point-holes for a given set of parameters. Two bridges are said to be homotopic to one another if one can be continuously distorted into the other without passing through a point-hole or having its endpoints leave the stable manifold(s). A family of bridges that are homotopic to each other is a "homotopy class." In Fig. 8, there are three bridges below the label $f^{t b}$ that (i) have one endpoint on $\mathbb{S}^{T}$ and one on $\mathbb{S}^{B}$, (ii) pass under the point-hole $H_{-1}^{B}$, and (iii) pass above the point-hole $H_{-3}^{T}$. Each of these bridges can be continuously distorted into the others, are therefore homotopic to each other, and are elements of the same homotopy class.

We give each homotopy class of bridges a symbol, e.g., $f^{t b}$ or $c_{2}^{t}$. If bridges in a homotopy class have opposite direction, they are said to be inverses of the other. We say a bridge surrounds a point-hole if the point-hole is inside the region bounded by the bridge and the stable manifold segment connecting the bridge's endpoints [49]. If a bridge in a homotopy class surrounds a point-hole, its forward iterate must surround the forward iterate of that point-hole, and its endpoints must move closer to the unstable fixed point(s) they are approaching. For example, the bridge $c_{1}^{t}$ in Fig. 8 surrounds the hole $H_{-2}^{B}$, so its forward mapping must surround the hole $H_{-1}^{B}$. We then say that the bridge class $c_{1}^{t}$ maps forward as
$M\left(c_{1}^{t}\right)=c_{2}^{t}$, which gives us a symbolic representation for the evolution of $c_{1}^{t}$ under one mapping. Similarly, the symbolic representation for the mapping of a bridge homotopic to $c_{t}^{1}$, but with opposite direction, is $M\left(\left[c_{1}^{t}\right]^{-1}\right)=\left[c_{2}^{t}\right]^{-1}$.

If we repeat this on $c_{2}^{t}$, which surrounds $H_{-1}^{B}$, we can see that its forward iterate must surround $H_{0}^{B}$. We also know that its endpoints will be on $\mathbb{S}^{T}$ between the rightmost endpoint of $c_{2}^{t}$ and $\mathbf{z}_{x}^{R}$. However, $H_{0}^{B}$ lies outside the complex; consequently, the forward mapping of $c_{2}^{t}$ intersects $\mathbb{S}^{B}$ two times. Since a bridge can only intersect $\mathbb{S}^{T}$ or $\mathbb{S}^{B}$ at its endpoints, the forward mapping of $c_{2}^{t}$ must be divided into three bridges as follows: (i) the first bridge has its first endpoint on $\mathbb{S}^{T}$ and second on $\mathbb{S}^{B}$, (ii) the second bridge has both endpoints on $\mathbb{S}^{B}$ and surrounds $H_{0}^{B}$, and (iii) the third bridge has its first endpoint on $\mathbb{S}^{B}$ and the final endpoint on $\mathbb{S}^{T}$. We say that the bridge class $c_{2}^{t}$ maps forward to a product of three bridge classes: $M\left(c_{2}^{t}\right)=f^{t b}\left[u_{0}^{b}\right]^{-1}\left[f^{t b}\right]^{-1}$. Furthermore, we have also uncovered the critical information generated by the theory: the bridge class $\left[u_{0}^{b}\right]^{-1}$ (and its inverse) lies outside the complex, so any bridge homotopic to $c_{2}^{t}$ (regardless of direction) will have a portion that escapes at the next iterate. In addition, we can also see that this portion will escape to the left of the barriers.

Similarly, bridge $c_{2}^{b}$ surrounds point-hole $H_{-1}^{T}$, and its forward mapping must surround $H_{0}^{T}$, which lies outside the complex. When mapped forward, the bridge class $c_{2}^{b}$ intersects $\mathbb{S}^{T}$ two times, and it must be divided into three bridge classes; we say that $M\left(c_{2}^{b}\right)=f^{b t}\left[u_{0}^{t}\right]^{-1}\left[f^{b t}\right]^{-1}$. The bridge class $\left[u_{0}^{t}\right]^{-1}$ (and its inverse) lies outside the complex, so any bridge homotopic to $c_{2}^{b}$ will have a portion escape at the next iterate, and this portion will escape to the right of the barriers.

\section{Additional bridges}

As mentioned earlier, an important difference between this problem and earlier ones [37-50] is that we are now examining a full scattering system, with particles approaching from and receding to infinite distance. (In previous work, we examined only "half-scattering," i.e., escape from a confined region.) A consequence is that the sets of bridges previously defined using only the unstable manifolds are no longer sufficient for describing our initial-condition lines.

To see why, we must study $L_{0}$ in detail. Figure 9 shows a qualitative $L_{0}$ of Type II [solid light gray (green online) curve], $\mathbb{S}^{T}, \mathbb{S}^{B}, S^{L}$, and $S^{R}$ (dotted curves; red online), and $\mathbb{U}^{T}, \mathbb{U}^{B}$, $U^{L}$, and $U^{R}$ [solid dark gray (blue online) curves]. The starting point of $L_{0}$ is labeled $\mathrm{A}$, and its endpoint is labeled $E$. One may follow $L_{0}$ from points $\mathrm{A}$ to $\mathrm{B}$ to $\mathrm{C}$ to $\mathrm{D}$ to $\mathrm{E}$.

In the past [49], we defined bridges as homotopy classes of unstable manifold segments with endpoints on the stable manifolds bounding the complex, $\mathbb{S}^{T}$ or $\mathbb{S}^{B}$. However, one can see that segments $\mathrm{AB}, \mathrm{BC}$, and $\mathrm{DE}$ of $L_{0}$ do not have both endpoints on $\mathbb{S}^{T}$ or $\mathbb{S}^{B}$. Each of these segments has at least one endpoint on $S^{L}$. Furthermore, no unstable manifold can intersect $S^{L}$ (if it did, the intersection would map to both an unstable fixed point and $x=-\infty$ as $t \rightarrow-\infty$ ). It follows that segments $\mathrm{AB}, \mathrm{BC}$, and $\mathrm{DE}$ cannot be homotopic to any bridges formed by segments of unstable manifolds. Hence, previous methods are not capable of fully describing the topological 


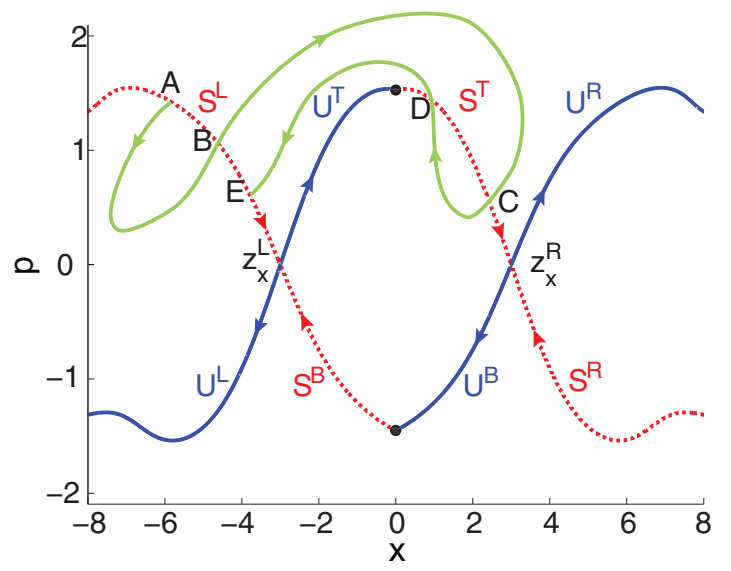

FIG. 9. (Color online) Qualitative graph showing a Type II $L_{0}$ [solid light gray (green) curve], $\mathbb{S}^{T}, \mathbb{S}^{B}, S^{L}$, and $S^{R}$ (dotted red curves), and $\mathbb{U}^{T}, \mathbb{U}^{B}, U^{L}$, and $U^{R}$ [solid dark gray (blue) curves]. Since no unstable manifold can intersect $S^{L}$, the $L_{0}$ segments $\mathrm{AB}$, $\mathrm{BC}$, and $\mathrm{DE}$ cannot be homotopic to any bridge formed by an unstable manifold.

structure of all segments of $L_{0}$ for the present system. Similar problems occur for $L_{0}$ of Type IV.

We must develop a more general definition of bridges. From Fig. 9 it is plain that we need an additional bridge or bridges that go from $S^{L}$ to $\mathbb{S}^{T}$, which are homotopic to segments BC and DE. We also need an additional bridge that begins and ends on $S^{L}$, and which lies "below" it, so it is homotopic to segment $\mathrm{AB}$. The endpoints of segment $\mathrm{AB}$ march toward the fixed point $\mathbf{z}_{x}^{L}$ as $L_{0}$ is mapped forward, and the segment stretches to the left and down as they do so. Since the desired bridge is the entire homotopy class for segment $\mathrm{AB}$, the bridge class will always map to itself, and it represents a segment of $L_{0}$ that is directly reflected, without entering the complex.

The same general arguments apply for $L_{0}$ representing particles approaching the barriers from the right. We need to include new bridges with endpoints on $S^{R}$. The types of new bridges that may be needed to accomodate $L_{0}$ can be summarized by the following. If particles approach from the left,

Type $S_{L L}$ : Both endpoints on $S^{L}$, lies "below" it.

Type $S_{L T}$ : One endpoint on $S^{L}$; one endpoint on $\mathbb{S}^{T}$.

If particles approach from the right,

Type $S_{R R}$ : Both endpoints on $S^{R}$, lies “above" it.

Type $S_{R B}$ : One endpoint on $S^{R}$; one endpoint on $\mathbb{S}^{B}$.

\section{Topological predictions}

We now have all the tools necessary to begin the topological analysis of initial-condition lines through the barrier region. We start by computing the stable manifolds and constructing the appropriate $L_{0}$. We then iterate $U_{0}^{B}$ and $U_{0}^{T}$ forward $J$ times and identify all $J$-neighbors along $S_{0}^{T}$ and $S_{0}^{B}$. These $J$-neighbor pairs are then mapped forward $J-k$ times and backward $k$ times, where $k$ is the transition number of the $J$-neighbors, which yields all $J$-neighbors for the chosen
$J$. We check for the existence of $J$-subneighbors along the stable manifold segment connecting every pair of $J$-neighbors. We then punch a point-hole in the phase plane in the appropriate place for each pair. We next determine all homotopy classes of bridges, including "additional" bridges which are not segments of unstable manifolds, give each a symbol, and develop an algebraic representation for how each symbol evolves.

Since the forward iterates of $L_{0}$ approach and stretch along unstable manifolds, $L_{0}$ evolves topologically in the same manner as the unstable manifolds. We develop a symbolic representation for $L_{0}$ by decomposing it into segments, each of which has endpoints on $\mathbb{S}^{B}, \mathbb{S}^{T}, S^{L}$, or $S^{R}$ and does not otherwise intersect those stable manifolds (as in Fig. 9). Each of these segments is homotopic to a bridge class, which allows us to symbolically express $L_{0}$ as a product of symbols. The mapping of curves induces a mapping of symbols. Each symbol can be mapped algebraically an arbitrary number of times, allowing us to express the $n^{\text {th }}$ forward iterate of $L_{0}, L_{n}$, by iterating each symbol in $L_{0} n$ times. Certain symbols in $L_{n}$ represent escape to the left of the barrier region $\left(u_{0}^{b}\right.$ and its inverse), and other symbols represent escape to the right of the barrier region ( $u_{0}^{t}$ and its inverse). The relative order of escape segments along $L_{0}$ is also predicted by this method.

The symbolic representation thus tells us the following: (i) the number of reflected escape segments that are forced to occur at each iterate, (ii) the number of transmitted escape segments that are forced to occur at each iterate, and (iii) their relative order along $L_{0}$. It should be noted that all three of these depend on the amount of initial topological information used and that starting with more initial toplogical information may yield additional predicted escape segments.

The initial topological structure of the heteroclinic tangle and $L_{0}$ forces certain topological properties to be present at later times. Homotopic lobe dynamics predicts all escape segments, and their relative order, that are forced to occur as a result of the initial topological information. Every predicted escape segment must be seen in computations, but there may be other escape segments that occur, which are not forced by the initial topological structure. In this sense, one may think of homotopic lobe dynamics as a method for finding the minimal number, and relative order, of escape segments forced to occur at each iterate by the initial topological structure. As previously mentioned, choosing to begin the analysis with more initial topological information, i.e., choosing a higher $J$, may allow additional escape segments to be predicted at later times.

In the next section, we implement the method discussed here on a specific case, the results of which were given in Sec. IV.

\section{CASE STUDY}

Recall that our system parameters are $\hat{x}=3, U_{0}=1, \alpha=$ $0.5, \omega=2 \pi / 3, \phi=\pi / 2, \sigma=1$, and $m=1$. We examine an initial-condition line of particles approaching the barriers from the left with initial momentum $p_{0} \approx 1.50$ (more precisely $p_{0} \approx 1.49959$ ).

Manifolds are constructed by iterating initial-condition lines that begin very close to, and lying on eigenvectors 


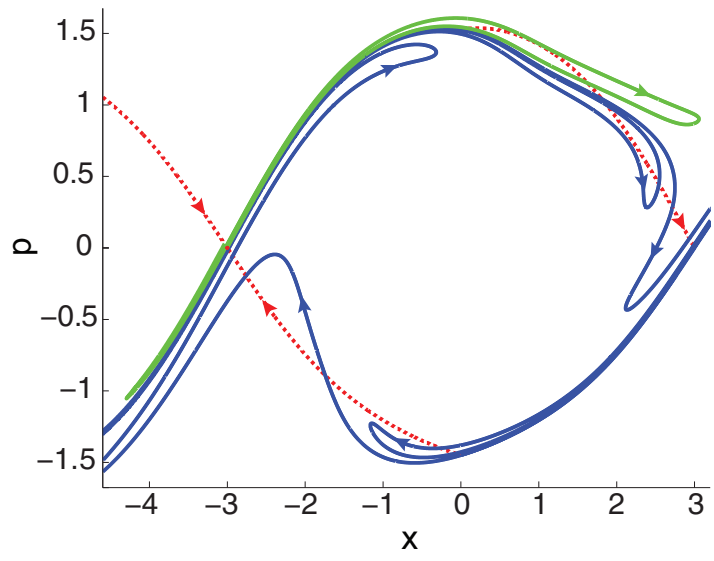

FIG. 10. (Color online) $\mathbb{S}^{T}, \mathbb{S}^{B}$, and $S^{L}$ [dotted (red) curves], $\mathbb{U}^{T}$, $\mathbb{U}^{B}$, and the first $J=3$ forward iterates of $U_{0}^{T}$ and $U_{0}^{B}$ [solid dark gray (blue) curves], and $L_{0}$ [solid light gray (green) curve], which is a forward iterate of $l_{0} . L_{0}$ is chosen at the earliest iterate to have an intersection with $\mathbb{S}^{T}$ and is of Type II. The endpoints of $L_{0}$ are on $S^{L}$ at the far left. The segment below $S^{L}$ forms a loop.

passing through, the unstable fixed points. Stable manifolds are calculated by iterating the appropriate initial-condition lines backward, and unstable manifolds are computed by iterating forward. We must choose a number of iterates, $J$, to iterate the fundamental unstable manifold segments to use as the basis for our analysis; for this example, we have chosen three iterates, i.e., $J=3$.

Examining $S^{B}, S^{T}$, and $S^{L}$, we see that our initial momentum $p_{0} \approx 1.50$ lies in Region III in Fig. 6. By the rules discussed earlier, we place one endpoint of $l_{0}$ on $S^{L}$ and give it a width of $\Delta x_{0}=p_{0} 2 \pi / m \omega$. This $l_{0}$ is the solid gray horizontal curve (green online). Due to its construction, at a certain forward iterate, this $l_{0}$ will evolve into a Type II $L_{0}$. We choose $L_{0}$ to be the earliest iterate at which $l_{0}$ interesects $\mathbb{S}^{T}$. This $L_{0}$ is shown in Fig. 10, and its qualitative topological structure can be seen in Fig. 9.

Figure 11 shows all $J$-neighbors along $S_{0}^{T}$ (a) and $S_{0}^{B}$ (b). In Fig. 11(a), the $J$-neighbors along $S_{0}^{T}$, which we call $(\alpha, \beta)$, are marked by large black dots. In Fig. 11(b), the $J$-neighbors along $S_{0}^{B}$, which we call $(\gamma, \delta)$, are marked by large gray dots (yellow online). Since both $(\alpha, \beta)$ and $(\gamma, \delta)$ have transition number $k=J=3$, all other pairs of $J$-neighbors can be found by mapping $(\alpha, \beta)$ and $(\gamma, \delta)$ backwards $k=3$ times. Figure 11(a) also shows the pair of $J$-neighbors $\left(\gamma^{-2}, \delta^{-2}\right)$, which are the pair $(\gamma, \delta)$ mapped backwards twice. The $J$-neighbors $\left(\alpha^{-2}, \beta^{-2}\right)$ shown in Fig. 11(b) are $(\alpha, \beta)$ mapped backwards twice.

We need to determine if each pair of $J$-neighbors (eight total) has any $J$-subneighbors in between them. To do this, we must determine if $L_{0}$ enters the domain-hole associated with each pair of $J$-neighbors. In this example, $L_{0}$ only enters domain-holes associated with the $J$-neighbor pair $(\gamma, \delta)$ and their mappings. Figure 12 shows a zoom of the $\left(\gamma^{-2}, \delta^{-2}\right)$ domain-hole, along with $L_{0}$. The $J$-neighbors $\left(\gamma^{-2}, \delta^{-2}\right)$ are represented by large black dots. Since $L_{0}$ intersects the stable manifold segment connecting the $J$-neighbors, we must identify and use $J$-subneighbors when puncturing point-holes
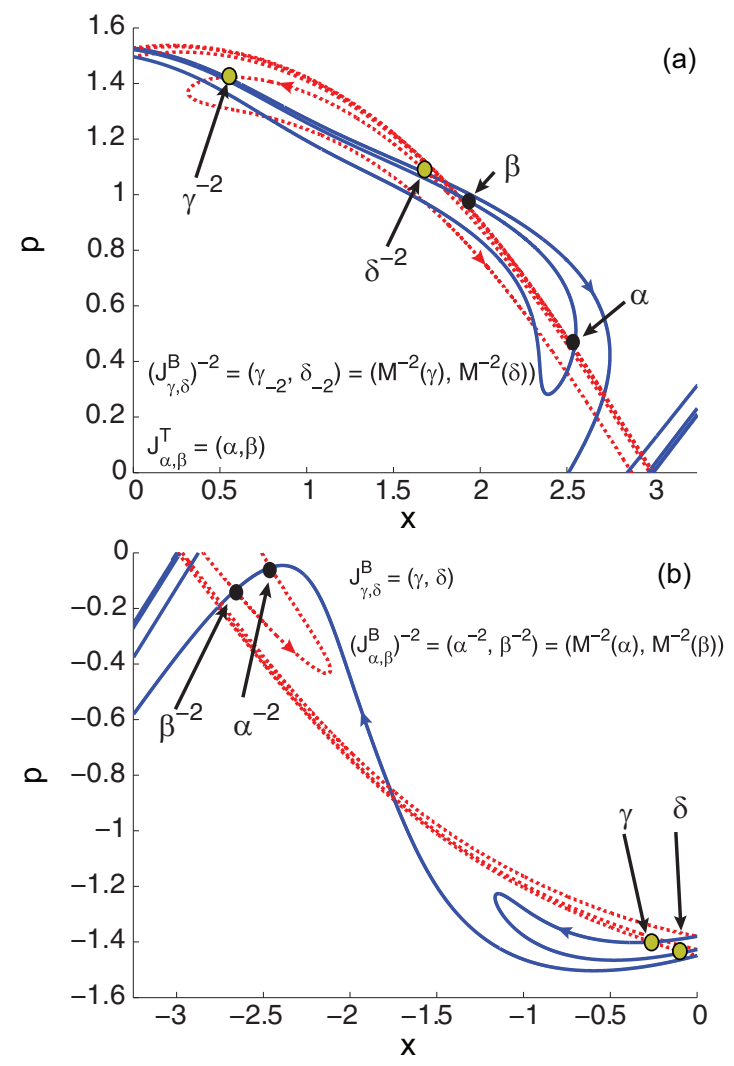

FIG. 11. (Color online) (a) Upper-right section of heteroclinic tangle. The black dots are $J$-neighbors along $S_{0}^{T}$, denoted $(\alpha, \beta)$. (b) Lower-left section of heteroclinic tangle. The gray (yellow) dots are $J$-neighbors along $S_{0}^{B}$, denoted $(\gamma, \delta)$. In (a), the gray (yellow) dots are the $J$-neighbors $\left(\gamma^{-2}, \delta^{-2}\right)$, which are $(\gamma, \delta)$ from (b) mapped backwards twice. In (b), the black dots are the $J$-neighbors $\left(\alpha^{-2}, \beta^{-2}\right)$, which are $(\alpha, \beta)$ from (a) mapped backwards twice.

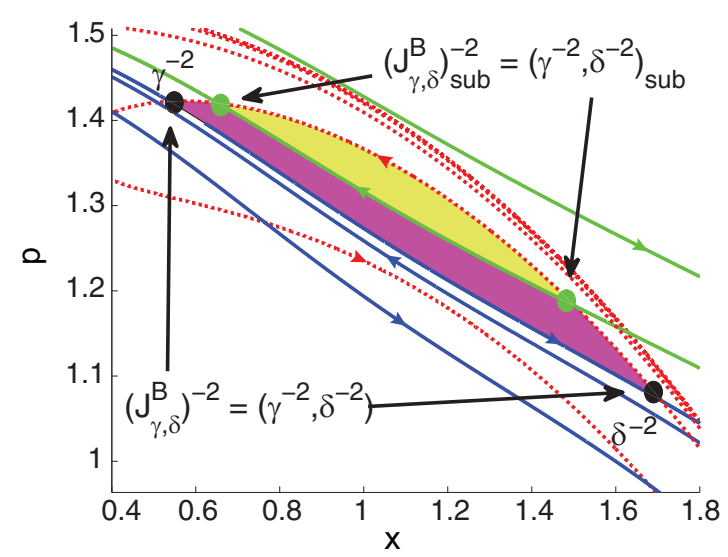

FIG. 12. (Color online) Zoom of the $J$-neighbors $\left(J_{\gamma, \delta}^{B}\right)^{-2}=$ $\left(\gamma^{-2}, \delta^{-2}\right)=\left(M^{-2}(\gamma), M^{-2}(\delta)\right)$. The $J$-neighbors are marked by the large black dots at the intersections between stable [dotted (red) curves] and unstable [solid dark gray (blue) curves] manifolds. This pair of $J$-neighbors has a set of $J$-subneighbors associated with it, which are represented by the large gray (green) dots. The domain-hole for the $J$-neighbor pair is colored in two shades of gray (yellow and pink), and the subhole associated with the $J$-subneighbor pair is the lighter gray (yellow) portion. 
in the plane for the series of holes associated with $(\gamma, \delta)$ and their mappings. The $J$-subneighbors are represented by large gray dots (green online) in Fig. 12. The entire shaded region is the domain-hole (yellow and pink online), and the subhole is the lighter gray portion (yellow online).

We must place a point-hole within the subhole associated with the $J$-neighbor pair $\left(\gamma^{-2}, \delta^{-2}\right)$, and arbitrarily close to to either of the $J$-subneighbors. The location of the point-hole should be within the lighter gray (yellow online) region of Fig. 12, and arbitrarily close to either of the gray (green online) dots. We then map this point-hole forward and backward the appropriate number of times, again puncturing a hole in the plane for each one.

No $J$-subneighbors exist between any of the $\left(M^{n}(\alpha), M^{n}(\beta)\right) J$-neighbors for this $L_{0}$, so we choose a point-hole inside the domain-hole associated with $(\alpha, \beta)$, and place it arbitrarily close to either $\alpha$ or $\beta$. We then map it backward $k=3$ times, puncturing a hole in the phase plane each time.

One must note that we are not necessarily free to place a point-hole arbitrarily close to every $J$-neighbor pair that does not have a pair of $J$-subneighbors along $L_{0}$ associated with it. If $L_{0}$ creates a pair of $J$-subneighbors between a pair of $J$-neighbors $(f, g)$, then $L_{1}$ will create a pair of $J$ subneighbors between the $J$-neighbors $(M(f), M(g))=(h, i)$. For this reason, one should not place a point-hole arbitrarily close to either $(h, i)$; instead, one must place the point-hole arbitrarily close to a $J$-subneighbor associated with $(f, g)$, and then map it forward and backward. This method ensures that all point-holes associated with this series of holes are placed in the appropriate location.

Bridges of the manifolds are shown in Fig. 8, and their homotopy classes are summarized here. Homotopy classes are determined according to bridge's relations to the punctured holes.

$f^{b t}$ : Starts on $\mathbb{S}^{B}$, ends on $\mathbb{S}^{T}$, passes below $H_{-3}^{B}$ and above $H_{-1}^{T}$, and immediately surrounds no domain-holes.

$f^{t b}$ : Starts on $\mathbb{S}^{T}$, ends on $\mathbb{S}^{B}$, passes below $H_{-1}^{B}$ and above $H_{-3}^{T}$, and immediately surrounds no domain-holes.

$c_{1}^{t}$ : Starts and ends on $\mathbb{S}^{T}$; surrounds $H_{-2}^{B}$.

$c_{2}^{t}$ : Starts and ends on $\mathbb{S}^{T}$; surrounds $H_{-1}^{B}$.

$u_{0}^{t}$ : Starts and ends on $\mathbb{S}^{T}$; surrounds $H_{0}^{T}$.

$u_{0}^{b}$ : Starts and ends on $\mathbb{S}^{T}$; surrounds $H_{0}^{B}$.

$c_{1}^{b}$ : Starts and ends on $\mathbb{S}^{B}$; surrounds $H_{-2}^{T}$.

$c_{2}^{b}$ : Starts and ends on $\mathbb{S}^{B}$; surrounds $H_{-1}^{T}$.

Figures 9 and 10 show that some segments of $L_{0}$ cannot be described by existing bridges, i.e., by segments of unstable manifolds. We need to create two new bridges for our analysis, with homotopy classes as follows:

$S_{L L}$ : Begins and ends on $S^{L}$, lies "below" it, and passes under $H_{B}^{-3}$.

$S_{L T}$ : Begins on $S^{L}$; ends on $\mathbb{S}^{T}$.

We have to choose an appropriate endpoint of $S_{L T}$ on $\mathbb{S}^{T}$ so segments of $L_{0}$ will be homotopic to it. We will choose $S_{L T}$

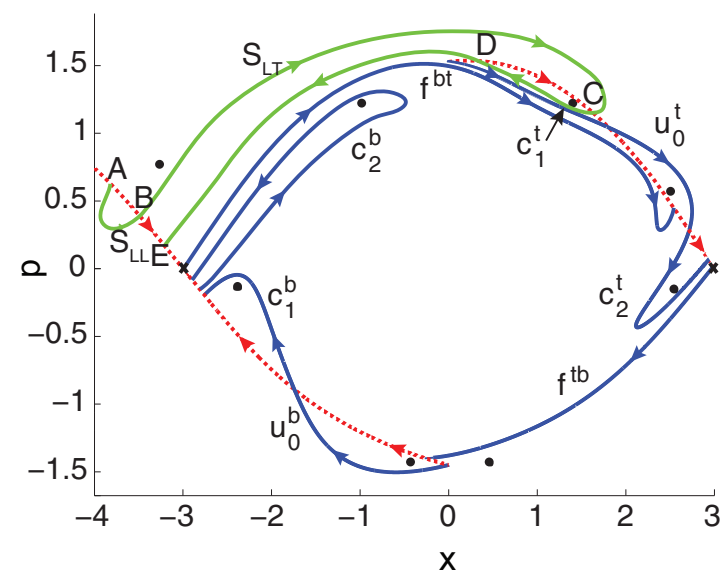

FIG. 13. (Color online) Qualitative rendering of $\mathbb{S}^{T}, \mathbb{S}^{B}$, and $S^{L}$ [dotted (red) curves], $L_{0}$ [solid light gray (green) curve], and all bridges necessary for the topological analysis for this example. The bridges $S_{L L}$ and $S_{L T}$ have at least one endpoint on $S^{L}$ and thus are not unstable manifold segments. All other bridges are unstable manifold segments [solid dark gray (blue) curves].

to be arbitrarily close to one of the segments of $L_{0}$ that goes from $S^{L}$ to $\mathbb{S}^{T}$.

Figure 13 shows a qualitative, but topologically correct, graph of $L_{0}$ [solid light gray (green online) curve] along with an example bridge from each homotopy class needed to carry out the topological analysis. The direction of $L_{0}$ is consistent with our chosen direction of $l_{0}$ (left to right). By construction, the endpoints lie on $S^{L}$. The starting point is labeled A. Segment AB is homotopic to $S_{L L}$. Segment BC is homotopic to bridge $S_{L T}$, which has a direction going from $S^{L}$ to $\mathbb{S}^{T}$. We choose $S_{L T}$ to lie arbitrarily close to segment $\mathrm{BC}$, and any $L_{n}$ segment with one endpoint on $S^{L}$, and the other endpoint between $P_{0}^{T}$ and $Q_{0}^{T}$ on $\mathbb{S}^{T}$, will be homotopic to $S_{L T}$ (or its inverse). Segment CD is homotopic to $\left(c_{1}^{t}\right)^{-1}$. Segment DE is homotopic to the inverse of $S_{L T}$.

Now the theory is complete, and we return to Sec. III. The symbols and mappings for these homotopy classes are given by Eq. (3). $L_{0}$ is expressed as a product of these symbols, given by Eq. (4). Each symbol in $L_{0}$ is algebraically mapped forward $n$ times, which gives the symbolic representation for $L_{n}$. The first few symbolic representations are given by Eqs. (6)-(8). Each instance of $u_{0}^{b}$ (or its inverse) or $u_{0}^{t}$ (or its inverse) in $L_{n}$ represents a segment that escapes to the left or right, respectively, of both barriers at the $n^{\text {th }}$ iterate. Comparison and discussion of predicted and computed escape segments for this case were given in Sec. IV.

Generalizing and extending HLD for use with full scattering systems has many purposes. In a ballistic atom pump, the primary goal is to calculate the net flow of particles that approach the barriers from both sides, and HLD allows one to use stable manifolds to place lower bounds on the number of particles which are transmitted or reflected by the barriers for all incoming energies (this is much faster than computing all trajectories). In this paper, HLD is primarily used to explain the fractal structure seen in escape-time graphs. In other contexts, HLD can also be used to find a minimal set of closed or periodic orbits $[45,51]$ in order to carry out semiclassical sums, 
to calculate topological entropy [48-50] (a measure of the complexity of the dynamics), and to partition mixed phase spaces [52].

\section{CONCLUSION}

We have shown how to extend homotopic lobe dynamics so it can be used to study a full scattering process with a heteroclinic tangle. We have shown how to select endpoints of the initial condition line, where to puncture point-holes in the phase plane, and that additional bridge types are necessary for the topological analysis. The methods outlined in this paper are very general and thus can be used for a large number of systems involving chaotic transport. Homotopic lobe dynamics can now be applied to full scattering systems in order to to calculate topological entropy [48-50], partition mixed phase spaces [52], find a minimal set of closed or periodic orbits [45,51], and sort interfering trajectories into groups for use in semiclassical theory.

This type of analysis allows one to make reliable predictions about some properties of a chaotic system at intermediate times, using topological properties of the system at early times. In this paper, we have shown that homotopic lobe dynamics both explains and predicts the fractal structure seen in escape- time graphs for a ballistic atom pump, i.e., for particles incident on two Gaussian-shaped potential barriers oscillating out-ofphase with one another. A future paper will present classical, semiclassical, and quantum theories of such pumps.

Eventually we hope that homotopic lobe dynamics can be automated, so numerical computations of stable and unstable manifolds can extract the symbolic dynamics and its predictions without human intervention. That advance will require knowledge of the application of homotopic lobe dynamics to many specific systems, such as the one considered here.

\section{ACKNOWLEDGMENTS}

This work was supported by the National Science Foundation under Grant No. 1068344. Some calculations in this work were performed on the SciClone computing complex at The College of William and Mary, which were provided with the assistance of the National Science Foundation, the Virginia Port Authority, and Virginia's Commonwealth Technology Research Fund. We also thank Kevin A. Mitchell of the University of California, Merced, and Seth Aubin and Megan K. Ivory of The College of William and Mary for many discussions of this work.
[1] K. K. Das and S. Aubin, Phys. Rev. Lett. 103, 123007 (2009).

[2] K. K. Das, Phys. Rev. A 84, 031601 (2011).

[3] T. A. Byrd, M. K. Ivory, A. J. Pyle, S. Aubin, K. A. Mitchell, J. B. Delos, and K. K. Das, Phys. Rev. A 86, 013622 (2012).

[4] R. W. Easton, Trans. Am. Math. 294, 719 (1986).

[5] M. J. Davis and S. K. Gray, J. Chem. Phys. 84, 5389 (1986).

[6] M. J. Davis, J. Chem. Phys. 83, 1016 (1985).

[7] R. S. MacKay, J. D. Meiss, and I. C. Percival, Physica D 13, 55 (1984).

[8] A. Tiyapan and C. Jaffé, J. Chem. Phys. 99, 2765 (1993).

[9] A. Tiyapan and C. Jaffé, J. Chem. Phys. 101, 10393 (1994).

[10] A. Tiyapan and C. Jaffé, J. Chem. Phys. 103, 5499 (1995).

[11] C. Jaffé, S. D. Ross, M. W. Lo, J. Marsden, D. Farrelly, and T. Uzer, Phys. Rev. Lett. 89, 011101 (2002).

[12] C. Jaffé, D. Farrelly, and T. Uzer, Phys. Rev. A 60, 3833 (1999).

[13] C. Jaffé, D. Farrelly, and T. Uzer, Phys. Rev. Lett. 84, 610 (2000).

[14] C. Jaffé and T. Uzer, Phys. Chem. A 105, 2783 (2001).

[15] S. Wiggins, L. Wiesenfeld, C. Jaffé, and T. Uzer, Phys. Rev. Lett. 86, 5478 (2001).

[16] V. Rom-Kedar, Physica D 43, 229 (1990).

[17] V. Rom-Kedar, Nonlinearity 7, 441 (1994).

[18] B. Eckhardt and C. Jung, J. Phys. A 19, L829 (1986).

[19] B. Eckhardt, J. Phys. A 20, 5971 (1987).

[20] C. Jung and H. J. Scholz, J. Phys. A 20, 3607 (1987).

[21] B. Rückerl and C. Jung, J. Phys. A 27, 55 (1994).

[22] B. Rückerl and C. Jung, J. Phys. A 27, 6741 (1994).

[23] C. Lipp and C. Jung, J. Phys. A 28, 6887 (1995).

[24] C. Jung, C. Lipp, and T. H. Seligman, Ann. Phys. 275, 151 (1999).
[25] C. Jung and A. Emmanouilidou, Chaos 15, 023101 (2005).

[26] A. Emmanouilidou and L. E. Reichl, Phys. Rev. A 62, 022709 (2000).

[27] A. Emmanouilidou and C. Jung, Phys. Rev. E 73, 016219 (2006).

[28] P. Collins, Internat. J. Bifur. Chaos Appl. Sci. Engrg. 12, 605 (2002).

[29] P. Collins, Dyn. Syst. 19, 1 (2004).

[30] P. Collins, Dyn. Syst. 20, 369 (2005).

[31] P. Collins, Experiment. Math. 14, 75 (2005).

[32] P. Collins and B. Krauskopf, Phys. Rev. E 66, 056201 (2002).

[33] E. A. Jackson, Perspectives of Nonlinear Dynamics (Cambridge University Press, Cambridge, 1990).

[34] S. Wiggins, Chaotic Transport in Dynamical Systems (Springer, New York, 1992).

[35] F. R. N. Koch, F. Lenz, C. Petri, F. K. Diakonos, and P. Schmelcher, Phys. Rev. E 78, 056204 (2008).

[36] M. Gärttner, F. Lenz, C. Petri, F. K. Diakonos, and P. Schmelcher, Phys. Rev. E 81, 051136 (2010).

[37] K. A. Mitchell, J. P. Handley, B. Tighe, J. B. Delos, and S. K. Knudson, Chaos 13, 880 (2003).

[38] K. A. Mitchell, J. P. Handley, J. B. Delos, and S. K. Knudson, Chaos 13, 892 (2003).

[39] K. A. Mitchell, J. P. Handley, B. Tighe, A. Flower, and J. B. Delos, Phys. Rev. Lett. 92, 073001 (2004).

[40] K. A. Mitchell, J. P. Handley, B. Tighe, A. Flower, and J. B. Delos, Phys. Rev. A 70, 043407 (2004).

[41] J. B. Delos and K. A. Mitchell, Few-Body Syst. 38, 181 (2006).

[42] P. Hansen, K. A. Mitchell, and J. B. Delos, Phys. Rev. E 73, 066226 (2006).

[43] C. Bracher and J. B. Delos, Phys. Rev. Lett. 96, 100404 (2006). 
[44] C. R. Schleif and J. B. Delos, Phys. Rev. A 76, 013404 (2007).

[45] K. A. Mitchell and J. B. Delos, Physica D 229, 9 (2007).

[46] K. A. Mitchell, J. P. Handley, B. Tighe, A. A. Flower, S. K. Knudson, and J. B. Delos, in From the Atomic to the NanoScale, Proceedings of the International Workshop, edited by $\mathrm{C}$. T. Whelan and J. H. McGuire (Old Dominion University Press, Norfolk, VA, 2003).

[47] J. Novick, M. L. Keeler, J. Giefer, and J. B. Delos, Phys. Rev. E 85, 016205 (2012).
[48] J. Novick and J. B. Delos, Phys. Rev. E 85, 016206 (2012).

[49] K. A. Mitchell and J. B. Delos, Physica D 221, 170 (2006).

[50] K. A. Mitchell, Physica D 238, 737 (2009).

[51] K. A. Mitchell, AIP Conf. Proc. 1468, 268 (2012).

[52] K. A. Mitchell, Physica D 241, 1718 (2012).

[53] See Supplemental Material at http://link.aps.org/supplemental/ 10.1103/PhysRevE.89.022907 for table of notations.

[54] C. Jung, C. Mejía-Monasterio, O. Merlo, and T. H. Seligman, New J. Phys. 6, 48 (2004). 